\title{
The role of separation of scales in the description of spray combustion
}

\author{
Antonio L. Sánchez ${ }^{\mathrm{a}, *}$, Javier Urzay ${ }^{\mathrm{b}}$, Amable Liñán ${ }^{\mathrm{c}}$

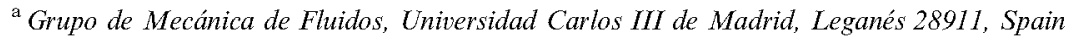 \\ ${ }^{\mathrm{b}}$ Center for Turbulence Research, Stanford University, Stanford, CA 94305-3024, United States \\ ${ }^{\mathrm{E}}$ ETSI Aeronáuticos, Pl. Cardenal Cisneros 3, Madrid 28040, Spain
}

\begin{abstract}
The present paper deals with the description of the interacting multiscale processes governing spray vaporization and combustion downstream from the near-injector atomization region in liquid-fueled burners. One of the main objectives is to emphasize the progress made in the mathematical description and understanding of reactive spray flows by incorporation of rationally derived simplifications based on the disparity of length and time scales present in the problem. In particular, we aim to show how the disparity of the scales that correspond - with increasing values of their orders of magnitude - to the droplet size, interdroplet spacing, and width of the spray jets, ensures the validity of their homogenized description. The two-way coupling associated with exchanges of mass, momentum, and energy between the gas and the liquid phases is dominated by the homogenized exchanges with the gas provided collectively by the droplets, and not by the direct interaction between neighboring droplets. The formulation is used as a basis to address nonpremixed spray diffusion flames in the Burke-Schumann limit of infinitely fast chemical reactions, with the conservation equations written in terms of chemistry-free coupling functions that allow for general nonunity Lewis numbers of the fuel vapor. Laminar canonical problems that have been used in the past to shed light on different aspects of spray-combustion phenomena are also discussed, including spherical spray clouds and structures of counterflow spray flames in mixing layers. The presentation ends with a brief account of some open problems and modeling challenges.
\end{abstract}

(

Keywords: Separation of scales; Spray combustion; Liquid-fueled propulsion; Multiphase flows

\section{Introduction}

The existence of length and time scales of very different magnitude is a complicating characteristic of many problems encountered in fluid mechanics and combustion. The mathematical description of the associated flows can be facilitated by accounting for the disparity of these scales. A renowned example of the success of this approach is the boundary-layer theory developed by Ludwig Prandtl over a century ago. Separation of scales has also been extensively used in connection with the description of combustion problems, where the disparity of time scales is often due to the strong temperature sensitivity of the chemical 
reactions. For spray combustion, additional length and time scales originate from the twophase nature of the flow.

Over the past half century combustion modelers have successfully exploited the separation of the scales present in the vaporization and combustion of droplets and sprays to generate simplified equations for the description of reactive spray flows. The purpose of this topical review lecture is to give an overview of the progress achieved and to describe some recent results. The presentation will begin with a discussion of the reasons for the validity, and also the shortcomings, of the continuum description of the gas and liquid phases in the vaporization and combustion of sprays. Because of the important role of the interphase exchange rates of mass, energy, and momentum, a summary of these rates is given, and then used in the conservation equations for the description of reacting sprays; this simplifies in the important extreme limiting cases of pure spray vaporization, without chemical reactions, and diffusion-controlled spray combustion. Simple laminar canonical problems, widely used in the past in fundamental investigations of spray combustion, are formulated in nondimensional form to identify the parameters that characterize the interplay of the different spray physicochemical phenomena.

Substantial research efforts have been made in the past in connection with the problems of vaporization and combustion of droplets and droplet arrays [1-9], ignition of fuel sprays [10], and dynamics and modeling of turbulent sprays [1113]. Related work on atomization of liquid jets [14 16] and on the dynamics of particle-laden turbulent flows [17-19] is relevant for understanding the generation and dispersion of sprays. A reference book including an updated comprehensive presentation of the current level of understanding of fluid dynamics and transport of droplets and sprays is available [20]. In addition, other relevant literature include reference textbooks on atomization [21] and multiphase combustion [22], as well as research monographs $[23,24]$.

The design of liquid-fueled combustion systems is subject to a number of constraints stemming from the need to vaporize the droplets, mix the fuel vapor with the surrounding air, and ignite and burn completely the resulting mixture in the limited available residence time, with the scales and parameters of these different physicochemical processes entering in the determination of the combustor performance. An important consideration that must be taken into account when describing vaporization and combustion in diesel engines, and also in the primary combustion zone of gas turbines, is the large value of the liquid-to-gas density ratio, on the order of a few hundred in many applications. Also relevant for combustion is the large value, of order $S \sim 15$, of the mass of air required to burn in stoichiometric proportions the unit mass of fuel. Another basic consideration pertaining to the required dispersion of the droplets in the combustion chamber is that the heat needed for the vaporization of each droplet comes from the sensible heat of the gas within the spray, so that vaporization in the bulk of the spray can only start when sufficiently dilute conditions are reached; otherwise the amount of gas entrained by the spray is insufficient to provide the heat of vaporization. In assessing the coupling between the liquid and gas phases, one must also bear in mind that the heat released by burning the fuel is enough lo lead to flame temperatures several times larger than the initial liquid temperature.

The large temperature sensitivity of the combustion reactions also enters in a fundamental way. For instance, in continuous-combustion devices this temperature sensitivity explains the onset of ignition near the hot boundary in mixing layers separating the spray from the preheated air. An important consideration, relevant for the selection of the atomizer in a given application, is that the droplet size must be small enough to ensure their complete vaporization and prevent their impingement with the confining walls. In view of the above considerations, it is clear that spray combustion stands out as a very particular category within the general field of two-phase flows, one that cannot be understood without accounting for its distinctive attributes.

The remainder of this paper is organized as follows. Some general comments concerning the specific characteristic of spray flows in combustion applications are given in Section 2, followed in Section 3 by a homogenized formulation for spray combustion that will serve as analytical framework for the rest of the paper. A qualitative description of spray combustion phenomena is presented in Section 4. The limit of infinitely fast chemical reaction is considered in Section 5, which provides a general Burke-Schumann formulation for the computation of spray flames. Sections 6 and 7 are devoted to the characterization of elementary spray structures. The final section outlines some of the open problems in spray combustion, including modeling issues.

\section{Preliminary considerations pertaining to spray flows in combustion systems}

\subsection{Atomization in spray-combustion applications}

The reduction in spray length required by the limited size of the combustion chamber can only be accomplished when there exists a significant velocity difference between the liquid jet or sheet to be atomized and the surrounding coflowing gas [21]. This is the case if the liquid stream is 
injected at high velocity, as in pressure atomizers of diesel engines, or by exposing the liquid to a high-velocity air stream, the method used in airassist and airblast atomizers. The hydrodynamic instabilities involved in the breakup process, different for pressure and airblast atomizers [14 $16,20,25]$, lead to primary atomization of the liquid vein to form ligaments and droplets, which further break up downstream as a result of the interactions with the surrounding turbulent gaseous flow in the secondary atomization region.

To guide the following discussion, a sketch of the spray flow corresponding to a plain-orifice atomizer, used in diesel combustion engines and turbojet afterburners, is shown in Fig. 1. In this kind of pressure atomizer, the liquid is injected from a high-pressure chamber through small circular holes, forming high-velocity jets that disintegrate rapidly downstream to form a jet spray with a small cone angle, whose value may depend on the transverse velocities induced inside the injector by the instabilities of the cavitating flow [26]. The working principle of other pressure atomizers can be more complex. For instance, in pressure-swirl atomizers, considered for instance in the schematic representation of a liquid-fueled combustor shown in Fig. 2, to be discussed later, the fuel is injected through tangential ports in the injector plenum. Because of angular-momentum conservation, the resulting swirling motion is transformed, near the injector orifice, into kinetic energy of the axial and radial motion of the liquid, generating a conical sheet that breaks up to give a hollow-cone spray $[27,28]$.

The design of the injection system must satisfy a number of constraints. To ensure that the penetration length $L_{s}$ of the spray is comparable to the cylinder radius in diesel engines - or to the size of the primary combustion zone in gas turbines - the injection velocity $U_{I}$ must be sufficiently large, while still giving time for the droplets to vaporize, thereby avoiding their collision with the walls of the combustor, an undesired phenomenon that would lead to increased unburnt hydrocarbon emissions. On the other hand, the orifice radius of the injector $R_{I}$ must be small to lead, after

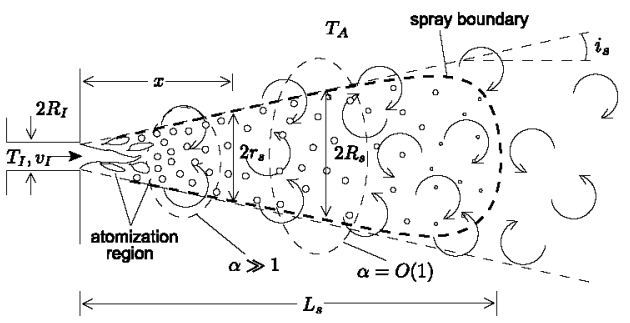

Fig. 1. A schematic view of a vaporizing spray generated by a plain-orifice pressure atomizer, including characteristic scales and regions. atomization of the liquid jet or of the conical sheet issuing from pressure-swirl atomizers, to droplets with sizes small enough that they vaporize and simultaneously penetrate a distance $L_{s}$, of the order of the chamber size, before reaching the combustor wall.

The distribution of droplet radii is determined by the phenomena occurring in the atomization region, where the spray is still dense and its velocity and temperature do not differ substantially from their injection values $U_{I}$ and $T_{I}$. In this atomization region, the droplets acquire a transverse velocity that, although small compared with $U_{I}$, is responsible for the initial transverse dispersion of the spray, which is further enhanced downstream by the turbulent motion acquired by the entrained air. The resulting spray jet is typically very slender. For instance, in plain-orifice atomizers, droplet dispersion results in small cone angles $2 i_{s}$ that usually lie between $5^{\circ}$ and $15^{\circ}$ [21]. Similar considerations apply also to the slender conical sprays produced by pressure-swirl atomizers or by liquid atomizers assisted by swirling air flows.

Direct numerical simulations can be instrumental in revealing the highly complex interactions occurring in the atomization region. Sample computations of the primary breakup region near the injection orifice are available [29-33]. Future improvements in computational capabilities will enable extended computations of both primary and secondary atomization regions in transient and stationary jet configurations to be performed, thereby providing quantitative information for the parametric dependence of the downstream jet-spray characteristics.

Droplet heating and vaporization can only occur downstream from the atomization region, once droplet dispersion causes the spray to become sufficiently dilute to allow for the droplets to receive significant energy from the entrained gas. As a consequence, the processes of liquid-jet atomization, leading to spray formation, and those of spray vaporization and combustion occur in separate spatial regions, which can be investigated independently. We shall focus below on the description of the different spray-combustion phenomena occurring in the dilute downstream region where the volume fraction occupied by the liquid phase is small, so that direct dropletdroplet interactions are unimportant, while the associated liquid mass loading is comparable to that of the entrained gas, as is needed to provide the heat required for droplet vaporization.

\subsection{Inter-phase coupling dynamics}

A measure of the monodisperse spray droplet population is the local number density $n$, i.e., number of droplets per unit volume, which decreases rapidly along the spray. Correspondingly, $l_{d}=n^{-1 / 3}$ is the characteristic interdroplet 


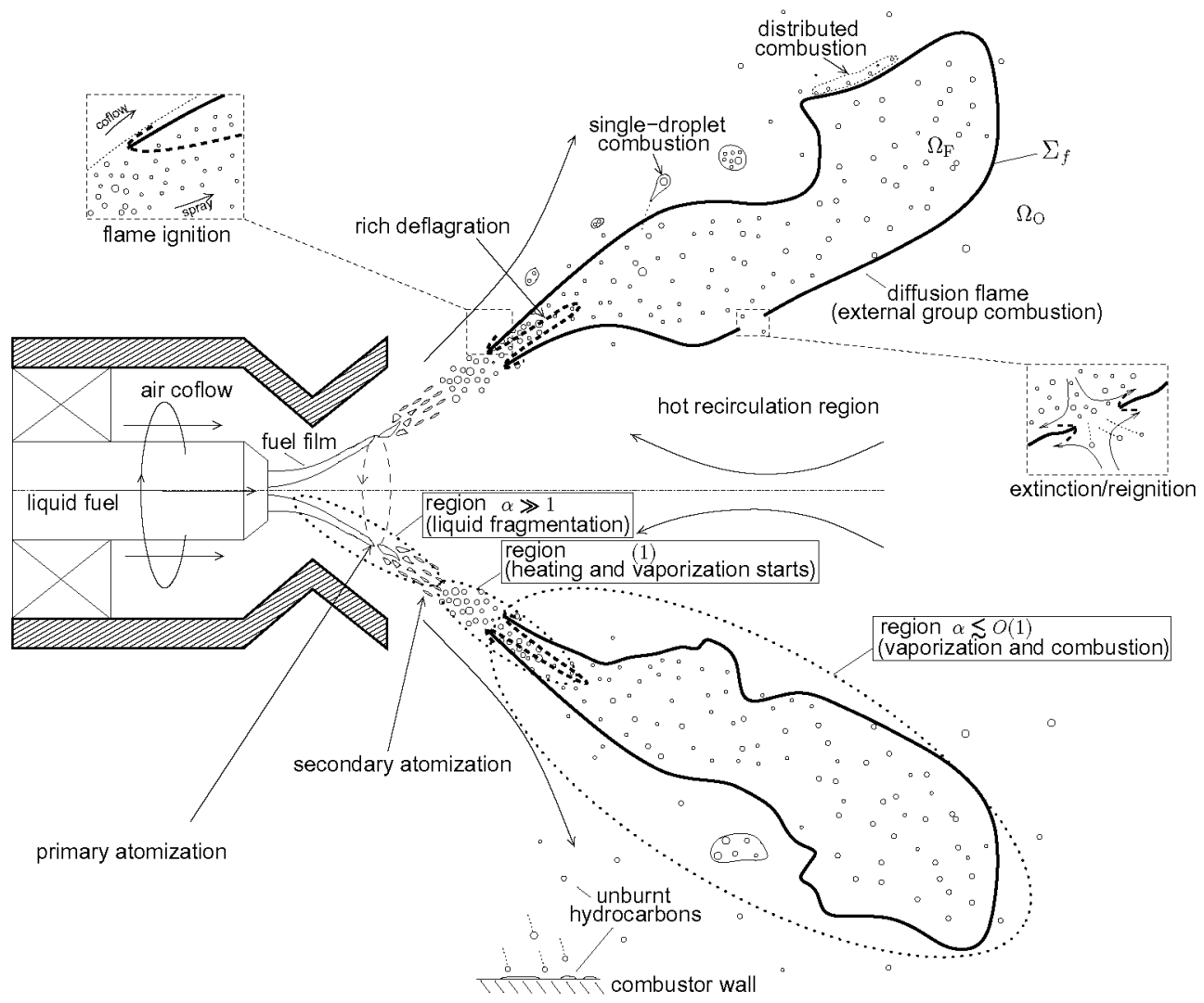

Fig. 2. Sketch of fundamental spray-combustion processes in typical liquid-fueled continuous-combustion systems.

distance, to be compared with the characteristic droplet radius $a$. We shall limit our analysis of the spray flow to dilute regions where the liquid volume fraction $\phi=(4 \pi / 3) a^{3} n$, of order $\left(2 a / l_{d}\right)^{3}$, is small, such that the interdroplet distance $l_{d}$ is significantly larger than $a$, resulting in negligible direct interaction between neighboring droplets. On average, each individual droplet can be envisioned as being centered in a cubic volume of gas of side length $l_{d}$. The ratio of the mass of the droplet, $(4 \pi / 3) \rho_{l} a^{3}$, to the mass of the corresponding surrounding gas, $\rho l_{d}^{3}(1-\phi)$, defines a mass-loading ratio $\alpha=(4 \pi / 3) a^{3} \rho_{l} n /[\rho(1-\phi)]$, where $\rho_{l}$ and $\rho$ represent the liquid and gas densities, respectively.

In most combustion systems, the gas and liquid densities are very different, the only exception being the conditions found in supercritical applications, for which $\rho \sim \rho_{l}$ in the vicinity of the droplets. For most of the other applications, the liquid-to-gas density ratio satisfies

$\frac{\rho_{l}}{\rho} \gtrsim 10^{2}$.

We shall see below that $\alpha$ is the fundamental parameter characterizing the collective inter-phase coupling for momentum and energy in spray flows, with effective two-way coupling occurring for values of $\alpha=O(1)$, corresponding to dilute sprays with small liquid volume fractions $\phi \sim \rho / \rho_{l} \ll 1$. Under these conditions the expression given above for $\alpha$ reduces to

$\alpha=\phi \rho_{l} / \rho=\frac{(4 \pi / 3) a^{3} n \rho_{l}}{\rho}=\frac{\pi}{6} \frac{\rho_{l}}{\rho}\left(\frac{2 a}{l_{d}}\right)^{3}$.

The distribution of $\alpha$ in the spray jet is nonuniform. Its value decreases due to droplet dispersion from very large values near the atomizer to small values sufficiently far downstream. A simple order-of-magnitude analysis for the spray jet formed in the plain-orifice atomizer of Fig. 1 serves to illustrate the downstream evolution of $\alpha$ in the bulk of the spray jet and its effect on the spray-jet development. In this case, the injection velocity $U_{I}$ and the contracted injector radius $R_{I}$ determine the injected liquid volume flow rate $\pi R_{I}^{2} U_{I}$. If, for simplicity, the spray formed upon atomization and breakup is considered to be monodisperse, with initial droplet radius $a_{o}$, then the number of droplets injected per unit time $\dot{N}$ from the atomization region into the combustion chamber is given simply by $\dot{N}=\left(\pi R_{T}^{2} U_{I}\right) /\left(\frac{4}{3} \pi a_{0}^{3}\right)$. The number of droplets is conserved before 
complete vaporization, so that the flux of droplets across the jet at a given downstream location must be equal to the number of droplets injected. In order of magnitude, this conservation condition leads to a first relationship

$n r_{s}^{2} u_{d} \sim \pi R_{I}^{2} U_{I} /\left(\frac{4}{3} \pi a_{o}^{3}\right)=\dot{N}$

linking, at an axial distance $x$, the characteristic value of $n$ with the corresponding values of the spray-jet radius $r_{s}$ and droplet axial velocity $u_{d}$. If the injector is discharging into air at rest, the momentum flux of the jet must be equal to the injection value $\pi R_{I}^{2} \rho_{l} U_{I}^{2}$, leading to a second relationship

$$
\left(\rho u^{2}+\rho_{l} \frac{4}{3} \pi a^{3} n u_{d}^{2}\right) r_{s}^{2} \sim \rho_{l} U_{I}^{2} R_{I}^{2},
$$

involving the characteristic axial component of the gas velocity $u$ as an additional quantity. In the initial region where $\alpha$ is still large compared with unity, the gas and droplet axial velocities $u$ and $u_{d}$ maintain a value close to $U_{I}$, imposed by the inertia of the liquid droplets. Droplet heating and vaporization are also negligible in this region because for $\alpha \gg 1$ the energy balance is dominated by the presence of the abundant cold liquid phase, with the result that the temperature of the entrained gas rapidly decreases to match the initial liquid temperature $T_{I}$, while the droplet temperature hardly increases. In the absence of droplet vaporization, the dispersion dynamics of the resulting two-phase jet can be expected to be in many respects identical to that observed in gaseous jets laden with solid particles. Due to air entrainment, the radius $r_{s}$ increases continuously with the downstream distance $x$ to the atomization region. If we consider for simplicity that the small spray angle $i_{s}$ is constant, so that $r_{s} \simeq i_{s} x$, then (3) and (4) lead to

$\phi=\alpha \rho / \rho_{l} \sim\left(R_{I} / r_{s}\right)^{2} \sim\left[R_{I} /\left(i_{s} x\right)\right]^{2}$

for the streamwise evolution of the liquid volume fraction.

As indicated in (4), the injected momentum flux, which is initially imparted to the droplets, is shared by the entrained air as the jet develops. In the initial region $\alpha \gg 1$ (although for a cubic array of droplets it is bounded by $\rho \alpha / \rho_{l}<\pi / 6$ ), and thus $u \simeq u_{d} \simeq U_{I}$, with most of the momentum flux still associated with the liquid phase. Significant droplet and gas deceleration starts to occur when the momentum flux of the entrained gas becomes comparable to that of the liquid phase, which, according to (4), occurs as the jet mass-loading ratio $\alpha$ decreases to values of order unity. It is also in this region, $\alpha=O(1)$, where significant droplet vaporization will occur in the combustion chamber.
In general, the liquid is initially cold and the heating and vaporization of the droplets rely on the sensible heat of the surrounding hot gas, which may include hot combustion products that recirculate in the combustion chamber and also preheated air. The extent of heat exchange between the liquid and gas phases depends on the local value of $\alpha$. Since the specific heat of the liquid fuel $c_{l}$ is comparable to the specific heat at constant pressure of the surrounding gas mixture $c_{p}$, significant liquid heating requires that the individual cold droplet be surrounded by a volume of hot gas of mass comparable to or larger than that of the droplet, corresponding to values of $\alpha$ of order unity or smaller. Likewise, droplet vaporization also necessitates $\alpha=O(1)$, because in practical applications the specific enthalpy of the hot gas is comparable to the latent heat of vaporization $L_{v}$. As a result, significant droplet heating and vaporization occur only when the mass loading ratio decreases to values of order unity.

According to (2) and (5), the mass-loading ratio $\alpha$ in the bulk of the spray decreases to values $\alpha=O(1)$ when the spray radius $r_{s}$ increases to large values of order

$R_{s}=\left(\frac{\rho_{l}}{\rho}\right)^{1 / 2} R_{I} \gg R_{I}$,

corresponding to large distances $x \sim L_{s} \sim R_{s} / i_{s}$, where a most important role in determining $L_{s}$ is played by the growth of $r_{s}$ associated with the gas entrainment. The design of the combustion system must ensure that the associated residence time $L_{s} / U_{I}$ is comparable to the characteristic droplet life time, defined below in (26), and also comparable to the characteristic ignition time. Under those conditions, droplet dispersion resulting from turbulent gas entrainment, droplet vaporization, and chemical reaction collaborate effectively to burn the spray.

\subsection{Collective effects in spray combustion}

As indicated in (1), in most combustion systems the liquid density is much larger than the characteristic gas density in the combustion chamber. As a consequence, in regions where $\alpha=O(1)$, which, as noted above, are the zones where spray heating, vaporization, and combustion start to occur, the characteristic interdroplet distance is significantly larger than the droplet diameter, i.e., $\quad l_{d} /(2 a) \sim\left[\rho_{l} /(2 \alpha \rho)\right]^{1 / 3} \gg 1$, which corresponds to large gas-to-liquid volume fractions $\phi^{-1}=\rho_{l} /(\alpha \rho) \gtrsim 10^{2}$ according to (1). These length scales are to be compared with the relevant macroscopic length scale $\ell$ of the problem (e.g., the thickness of the spray), which in most configurations of interest satisfies the condition $\ell \gg l_{d}$. For instance, for the slender jet spray of Fig. 1, 
the relevant macroscopic length $\ell$ is the characteristic radius $R_{s}$ corresponding to the region where the average mass loading ratio decreases to values of order unity, given in order of magnitude in (6). Using this last expression together with the condition $\alpha=O(1)$ provides $R_{s} / l_{d} \sim\left(R_{I} / a\right)\left(\rho_{l} / \rho\right)^{1 / 6}$. In typical plain-orifice atomizers, with values of $R_{I}$ on the order of a fraction of a millimeter, values of $a$ on the order of a a few tens of microns, and large values of $\rho_{l} / \rho$ in the range indicated in (1), the condition $R_{s} \gg l_{d}$ clearly holds.

In most systems, therefore, the characteristic scales of the problem satisfy

$\ell \gg l_{d} \gg a$.

Because of the condition $a \ll l_{d}$, each droplet vaporizes and moves without significant direct effects from neighboring droplets. The main effects on the vaporization of the droplets are not due to the direct influence of their neighbors, but are associated instead with their interaction with the mean gas-phase collective environment created by all the droplets. This is clearly the case in the important distinguished regime when the droplet Reynolds number $\mathrm{Re}_{d}$ (based on the droplet diameter, $2 a$, and the slip velocity, $\left|\mathbf{v}-\mathbf{v}_{d}\right|$, between the droplet and the local mean gas environment) is of order unity. Then, each droplet produces in the gas relatively large variations of the composition and temperature that are felt only in the immediate vicinity of the droplet, decaying rapidly at distances of the order of $a$, although more slowly in their wake, where the exchanges of mass, energy, and momentum between the droplet and gas are incorporated, in such a way that in most of the gas phase between droplets the variations of the gas properties are much smaller. The vaporization rate of and the force acting on each individual droplet are to be computed as those of the isolated droplet moving quasi-steadily, with the slip velocity, in the mean local environment. The description of the slow variations of the different gasphase variables, including the velocity, temperature, density, and relevant mass fractions, which occur over distances $\ell$ much larger than $l_{d}$, can be obtained at any spatial point by space-averaging over a neighborhood of that point of size $d$, with $d$ in the range $\ell \gg d \gg l_{d}$. Since $d^{3} \gg l_{d}^{3}$, each averaging cell includes many droplets, so that the corresponding point sources can be homogenized, as if they were homogeneously distributed, giving source terms that are proportional to the number of droplets per unit volume $n$. A noteworthy result of this homogenization process is that the intermediate length scale $l_{d}$ only appears indirectly in the formulation, with the resulting value of $n$ as a factor in the sources.

In the distinguished regime $\operatorname{Re}_{d} \sim 1$, both molecular transport and convective transport contribute to the droplet-gas exchange rates and, after dumping these exchanges in the wakes, to uniformize the local mean properties between the droplets. These exchanges involve a diffusion time $a^{2} / D_{T}$, where $D_{T}$ denotes the gas thermal diffusivity, and a residence time $a /\left|\mathbf{v}-\mathbf{v}_{d}\right|$, of the same order, both small compared with the characteristic droplet vaporization time $t_{v} \sim\left(a^{2} / D_{T}\right)$ $\left(\rho_{l} / \rho\right)$, defined below in (26). Clearly, the wakes of the droplets randomly located upstream of each droplet, representing the mean convective transport, and the transverse diffusion both cooperate to uniformize the interdroplet atmosphere. Therefore, within the averaging cell, the gas properties can be taken as uniform. The pronounced gasproperty changes occurring in the vicinity of each individual droplet (i.e., at distances of order $a$ ) can be neglected in the first approximation in the homogenized description, because the near-droplet regions occupy a negligible fraction $\phi \ll 1$ of the volume of the averaging cell. As discussed below in Section 3.2, the main effects of the local fluctuations emerging within the averaging cell as a result of the presence of the droplet wakes are included in the sources of the homogenized description.

While a single macroscopic scale $\ell$ can be often identified for laminar flows, a range of flow scales emerges in connection with the turbulent flow conditions found in practical applications, so that assessing the applicability of the criterion (7) is not straightforward. The integral scales of the turbulent flow, associated with the largest eddies, are comparable to, although somewhat smaller than, the macroscopic scales of the jet. For instance, in the main vaporization region $\alpha=O(1)$ of the plain-orifice configuration of Fig. 1, the size of the large eddies $\ell^{\prime}$ is a fraction of the spray radius $R_{s}$ and their associated velocity fluctuations $v^{\prime}$ are a fraction of the jet velocity $U_{I}$, giving eddy turnover times of order $\ell^{\prime} / v^{\prime} \sim R_{s} / U_{I}$. Clearly, the condition $R_{s} \gg l_{d} \gg a$ guarantees the validity of the multicontinua approach for the description of the interphase interactions occurring at these integral scales, including in particular the dispersion of the droplets, which is often dominated in shear flows by the large energetic eddies [34], because their turnover time $\ell^{\prime} / v^{\prime} \sim R_{s} / U_{I}$ is of the order of or larger than the droplet acceleration time $t_{a} \sim\left(a^{2} / D_{T}\right)\left(\rho_{l} / \rho\right)$.

These large eddies coexist and interact with smaller vortices, of decreasing size down to the Kolmogorov length scale $\ell_{k}$. Strictly speaking, the formulation given below applies to the direct numerical simulation of spray flows only when $\ell_{k} \gg l_{d}$, because for $\ell_{k} \sim l_{d}$ the averaging cell would be larger than the Kolmogorov length scale. However, the effects of the interaction of the droplets with the eddies of size below $\ell^{\prime}$ can be expected to be weak, because their turnover time is typically much smaller than the droplet acceleration time, with the result that the droplets behave ballistically with respect to the smallest 
eddies. In other words, the small, rapid velocity fluctuations of the Kolmogorov eddies, which modify the instantaneous slip velocity seen by the droplet, do not change appreciably the associated drag force, whose value is determined instead by the average slip velocity, with leading-order corrections to the motion transverse to the spray arising mainly from the largest eddies. In that sense, the multicontinua description can be expected to reproduce the main features of turbulent reacting sprays even when $\ell_{k} \sim l_{d}$, a condition often encountered in applications.

\section{Multicontinua formulation of reactive dilute sprays}

The disparity of scales typically present in practical spray-combustion applications enables a simplified description of the resulting flow to be performed in which the gas and liquid phases are treated as continuum media, whose evolution is coupled through the inter-phase exchange of mass, momentum, and energy [20]. The multi-continua formulation corresponding to a reactive polydisperse spray with $N_{c}$ different droplet classes is given below, including the separate sets of equations needed to describe the homogenized gas phase and the evolution of each droplet class. To complete the formulation, expressions are provided for the different droplet source terms.

\subsection{Conservation equations}

We begin by writing the homogenized gasphase conservation equations, which include the continuity, species, and momentum equations

$\frac{\partial \rho}{\partial t}+\nabla \cdot(\rho v)=\sum_{j=1}^{N_{c}} n^{j} \dot{m}^{j}$

$\frac{\partial}{\partial t}\left(\rho Y_{i}\right)+\nabla \cdot\left(\rho \mathbf{v} Y_{i}\right)+\nabla \cdot\left(\rho Y_{i} \mathbf{V}_{i}\right)=\dot{w}_{i}+\left\{\begin{array}{l}\sum_{j=1}^{N_{c}} n^{j} m^{j}(\text { if } i=\mathrm{F}), \\ 0(\text { if } i \neq \mathrm{F})\end{array}\right.$,

$\frac{\partial}{\partial t}(\rho \mathbf{v})+\nabla \cdot(\rho \mathbf{v})=\nabla \cdot \bar{\tau}-\nabla p^{\prime}+\sum_{j=1}^{N_{c}} n^{j}\left(\dot{m}^{j} \mathbf{v}_{d}^{j}-\mathbf{f}^{j}\right)$,

where $\mathbf{v}$ and $\bar{\tau}$ denote, respectively, the gas velocity and the viscous stress tensor. The gas composition is described in terms of the mass fraction $Y_{i}$ of the $N_{s}$ chemical species present in the mixture, with $\dot{w}_{i}$ representing the mass of species $i$ generated by chemical reaction per unit volume per unit time. Note that, since the sum of the $N_{s}$ conservation equations (9) leads to (8), the description of the gas phase requires the integration of $N_{s}-1$ of the conservation equations for the chemical species, the mass fraction of the $N_{s}$ th species (often $\mathrm{N}_{2}$ ) being computed from the identity $\sum_{i=1}^{N_{s}} Y_{i}=1$. For the conditions found in most spray-combustion applications, the prevailing Mach number is small, so that the relative spatial pressure variations are negligible. This is taken into account in the formulation by using in the momentum equation the variable $p^{\prime}=p-p_{o}(t)$, defined as the difference of the pressure from the value $p_{o}(t)$ found at a fixed arbitrary point in the combustor, the ratio $p^{\prime} / p_{o}$ being a small quantity of the order of the Mach number squared.

The summations appearing on the right-hand sides of the above equations are the coupling terms accounting for the presence of the droplets, which appear as distributed sources. In the formulation, the expressions written for these source terms correspond to a continuum description of the liquid phase, with the superscript $j$ used to indicate the properties of each one of the $N_{c}$ droplet classes considered. Thus, the continuity equation (8) and the fuel-vapor conservation equation include the mass of fuel vapor produced per unit volume per unit time, $\sum_{j=1}^{N_{c}} n^{j} \dot{m}^{j}$, with $n^{j}$ and $\dot{m}^{j}$ representing the number of droplets per unit volume and the rate of vaporization of the individual droplet. Similarly, momentum exchange between the liquid and gas phases is accounted for in writing $(10)$, where $\mathbf{v}_{d}^{j}$ is the average droplet velocity and $\mathbf{f}^{j}$ is the force of the gas on the individual droplet. Note that, if a Lagrangian description is employed instead for the liquid phase, then the source terms in the gas-phase equations would be calculated by evaluating the separate contributions of the different droplets present in each computational cell.

In the low-Mach-number limit, viscous dissipation can be neglected along with spatial pressure variations when writing the energy equation, thereby yielding

$$
\begin{aligned}
& \frac{\partial}{\partial t}(\rho h)+\nabla \cdot(\rho \mathbf{v} h)=-\nabla \cdot \mathbf{q} \\
& \quad-\sum_{j=1}^{N_{c}} n^{j}\left[\dot{m}^{j}\left(L_{v}-h_{\mathbf{F}_{s}}^{j}\right)+\dot{q}_{d}^{j}\right]+\frac{\mathrm{d} p_{o}}{\mathrm{~d} t},
\end{aligned}
$$

where $h=\sum_{i=1}^{N_{s}} Y_{i} h_{i}=\sum_{i=1}^{N_{s}} Y_{i}\left(h_{i}^{o}+h_{i}^{T}\right)$ is the gas enthalpy, expressed here for a mixture with $N_{s}$ different chemical species, with $h_{i}, h_{i}^{o}, h_{i}^{T}=\int^{T} c_{p_{i}} \mathrm{~d} T$, and $c_{p_{i}}$ representing the enthalpy, enthalpy of formation, thermal enthalpy, and specific heat at constant pressure of species $i$. The time variation of the pressure has been retained in (11), as it can be of importance for combustion in reciprocating engines. The expression for the heat-flux vector $\mathbf{q}=-\kappa \nabla T+\rho \sum_{i=1}^{N_{s}} h_{i} Y_{i} \mathbf{V}_{i}+\mathbf{q}_{R}$ includes the Fourier heat-conduction term $-\kappa \nabla T$, where $\kappa$ is the thermal conductivity and $T$ is the gas temperature, along with the radiative heat flux $\mathbf{q}_{R}$ and the energy transferred by diffusive transport of chemical species, $\rho \sum_{i=1}^{N_{s}} h_{i} Y_{i} \mathbf{V}_{i}$, where $\mathbf{V}_{i}$ is the diffusion velocity of species $i$. The droplet source term in (11) accounts for the heating and vaporization of the liquid fuel, with $\dot{q}_{d}^{j}$ being the heating rate of the individual droplet and 
$h_{\mathrm{F}_{\mathrm{s}}}^{j}=h_{\mathrm{F}}^{o}+\int^{T^{j}} c_{p_{\mathrm{F}}} \mathrm{d} T$ representing the enthalpy of the fuel vapor at the droplet-surface temperature $T_{d}^{j}$. It is worth noting that the effect of the chemical reactions on the energy balance is clearly apparent when the summation over all species of the enthalpy of formation $h_{i}^{o}$ times (9) is subtracted from (11) to give

$$
\begin{aligned}
\frac{\partial}{\partial t}\left(\rho c_{p} T\right)+\nabla \cdot\left(\rho \mathbf{v} c_{p} T\right)-\nabla \cdot(\kappa \nabla T) \\
=-\nabla \cdot \mathbf{q}_{R}-\sum_{i=1}^{N_{s}} h_{i}^{o} \dot{w}_{i} \\
\quad-\sum_{j=1}^{N_{c}} n^{j}\left[\dot{m}^{j}\left(L_{v}-c_{p} T_{d}^{j}\right)+\dot{q}_{d}^{j}\right]+\frac{\mathrm{d} p_{o}}{\mathrm{~d} t} .
\end{aligned}
$$

which is written explicitly in terms of the temperature $T$ by neglecting differences of specific heats $c_{p_{i}}$ from the mean value $c_{p}$.

The above equations for the gas and liquid phases must be supplemented with the equation of state $p_{o}=\rho R^{o} T \sum_{i=1}^{N_{s}}\left(Y_{i} / M_{i}\right)$, where $R_{-1}^{o}$ is the universal gas constant and $\left(\sum_{i=1}^{N_{s}} Y_{i} / M_{i}\right)^{-1}$ is the mean molecular mass of the gas mixture, with $M_{i}$ representing the molecular mass of species $i$. Also, appropriate constitutive equations must be given for the viscous stress tensor $\bar{\tau}$ and for the diffusion velocities, the latter often expressed in terms of Fick's law $\mathbf{V}_{i}=-D_{i} \nabla Y_{i}$, with $D_{i}$ representing the diffusion coefficient of the species $i$ into the gas mixture.

While a Eulerian description emerges naturally for the gas phase, the liquid phase is in principle more easily described with a Lagrangian approach in which each droplet is traced individually, with the ambient properties changing as the droplet moves across the flow field. The position of the droplet $k$ is determined by integrating the kinematic equation

$\frac{\mathrm{d} \mathbf{x}_{d}^{k}}{\mathrm{~d} t}=\mathbf{v}_{d}^{k}$.

Correspondingly, the evolution of the droplet velocity, $\mathbf{v}_{d}^{k}$, droplet radius $a^{k}$, and droplet temperature $T_{d}^{k}$ is obtained by integrating along the droplet trajectory the evolution equations

$$
\begin{aligned}
& \frac{4}{3} \pi \rho_{l}\left(a^{k}\right)^{3} \frac{\mathrm{d} \mathbf{v}_{d}^{k}}{\mathrm{~d} t}=\mathbf{f}^{k}, \\
& \frac{4}{3} \pi \rho_{l} \frac{\mathrm{d}\left(a^{k}\right)^{3}}{\mathrm{~d} t}=-\dot{m}^{k}, \\
& \frac{4}{3} \pi \rho_{l}\left(a^{k}\right)^{3} c_{l} \frac{\mathrm{d} T_{d}^{k}}{\mathrm{~d} t}=\dot{q}_{d}^{k} .
\end{aligned}
$$

In writing (16), the temperature is assumed to be uniform inside the droplet, a valid approximation when the heat conduction in the liquid droplet (possibly assisted by internal convection) is sufficiently fast for the associated conduction time to be much smaller than the droplet heating time $t_{q}$ from the injection temperature $T_{I}$ to a value close to the boiling temperature. Using the expression $t_{q}=\frac{1}{3}\left(c_{l} / c_{p}\right)\left(\rho_{l} / \rho\right)\left(a^{2} / D_{T}\right)$ given after (27) together with the estimate $a^{2} /\left[\pi \kappa_{l} /\left(\rho_{l} c_{l}\right)\right]$ for the heat conduction time through the droplet (including in the denominator a factor $\pi$ taken from the solution of heat conduction in the sphere [35]) indicates that this approximation of uniform droplet temperature is accurate when the thermal conductivity of the liquid fuel $\kappa_{l}$ is much larger than the gas thermal conductivity $\kappa$ [36], a condition satisfied in most situations (e.g., for methanol, $\kappa_{l} \simeq 0.2 \mathrm{~W} /(\mathrm{mK})$ at its boiling temperature, whereas for air $\kappa \simeq 0.03 \mathrm{~W} /(\mathrm{mK})$ at that same temperature). Clearly, this approximation of uniform droplet temperature becomes even more accurate in the presence of internal liquid circulation. For liquid fuels of low thermal conductivity equation (16) must be replaced during the fairly short heating period by a more complicated heating description accounting for the nonuniform temperature distribution inside the droplets [36], with consideration of the presence of recirculating liquid flow needed for increased accuracy [37].

In modeling combustion systems, the droplets are often introduced in the flow field at different entry ports, as required to mimic the injection characteristics of the system. Integrating with the given initial conditions provides the instantaneous distributions of $a^{k}, \mathbf{v}_{d}^{k}$, and $T_{d}^{k}$ along the droplet trajectories. In order to simplify the Lagrangian description, the droplets may be classified into classes, according to their origin and velocity of penetration at the entrance surface to the computational domain. The computation is coupled to that of the gas phase through the source terms in (8)-(11), which are to be evaluated in the numerical integration by accounting for the trajectories that traverse each averaging cell at a given time. This kind of tracking techniques is commonly employed in the particle-source-in-cell model [38] of typical turbulent combustion codes. Applications of this combined Eulerian-Lagrangian modeling strategy can be found, for instance, in computations of group combustion in pulverized coal furnaces [39-41] and of full-scale aeronautical combustors [42-44]. It should be noted, however, that the Lagrangian treatment of the liquid phase can lead to high computational costs and load-balancing issues in parallel computations with large numbers of droplets.

An alternative to this Eulerian-Lagrangian description is the so-called multi-continua formulation, in which the liquid phase is also treated as a continuum field including $N_{c}$ different droplet classes, with the droplet population of each droplet class $j$ described in terms of the number of droplets per unit volume $n^{j}$ through the conservation equation 


$$
\frac{\partial n^{j}}{\partial t}+\nabla \cdot\left(n^{j} \mathbf{v}_{d}^{j}\right)=0
$$

Correspondingly, in this Eulerian description the evolution equations along the trajectories (14)(16) are expressed for each droplet class with use made of the Eulerian differential operator $\mathrm{d} / \mathrm{d} t=\partial() / \partial t+\mathbf{v}_{d}^{j} \cdot \nabla()$. This continuum formulation is often simpler than formulations involving tracking of individual droplets and greatly facilitates analyses of laminar sprays. However, the continuum description of the liquid phase is known to be inadequate for addressing inertial sprays with multiple crossings of droplet trajectories, as occurs, for example, in turbulent flows or in the presence of recirculating flow regions when the particle size is not small enough. Although for some laminar flow configurations, such as those considered in Sections 6 and 7, introduction of specific modifications to the formulation renders the Eulerian description valid, methods based on Lagrangian descriptions of the liquid phase are in general needed in the presence of droplet crossings. A promising alternative approach for tackling the crossing of droplet trajectories in inertial sprays, based on field formulations of the liquid phase, employs quadrature methods of moments to solve the kinetic spray equation [45]. Despite the significant progress made recently in the development of these formulations [46,47], more work remains to be done to warrant full applicability to spray-combustion problems [48].

\subsection{Droplet submodels}

To complete the formulation, expressions must be provided for the exchange rates $\dot{m}, \dot{q}_{d}$, and $\mathbf{f}$ between the gas and the droplets. In the calculation, one needs to describe the flow field in the vicinity of the droplet, where the solution is quasisteady, as indicated before, because the associated diffusive transport time based on the droplet radius $a^{2} / D_{T}$ and the local residence time $a /\left|\mathbf{v}-\mathbf{v}_{d}\right|$ based on the slip velocity $\mathbf{v}-\mathbf{v}_{d}$, which we can consider to be of the same order, are always much smaller than the characteristic droplet life time $t_{v} \sim\left(a^{2} / D_{T}\right)\left(\rho_{l} / \rho\right)$, given below in (26), a result of the disparity $\rho_{l} \gg \rho$ [49]. Two main nondimensional parameters play a significant role in determining the near-droplet distributions of temperature and fuel mass fraction. One is the Reynolds number,

$\operatorname{Re}_{d}=\frac{\rho\left|\mathbf{v}-\mathbf{v}_{d}\right| 2 a}{\mu}$

defined in terms of the droplet diameter $2 a$ and the values of the gas density and viscosity corresponding to the surrounding atmosphere, $\rho$ and $\mu$. The other, an eigenvalue of the problem, is the nondimensional vaporization rate

$$
\lambda=\frac{\dot{m}}{4 \pi \kappa a / c_{p}},
$$

which represents a Stefan-flow Peclet number based on the mean radial gas velocity at the droplet surface $\dot{m} /\left(4 \pi a^{2} \rho\right)$. The value of $\lambda$ is determined by the balance between convective transport and conductive and diffusive transport of heat and species, associated with the temperature and concentration differences between the gas and the droplet surface, which are represented by the Spalding transfer numbers $c_{p}\left(T-T_{d}\right) / L_{v}$ and $\left(Y_{\mathrm{F}, S}-Y_{\mathrm{F}}\right) /\left(1-Y_{\mathrm{F}, S}\right)$, where $Y_{\mathrm{F}, S}$ is the fuel-vapor mass fraction at the droplet surface. As seen below, for low Reynolds numbers, $\lambda$ is given by the algebraic equations (23) and (24).

In combustion applications, the resulting values of $\lambda$ range, as we shall see below, from small, in the initial stages of droplet heating, to values at most of order unity during the main vaporization stage (note that the nondimensional vaporization rate $\lambda$ can never be much larger than unity, because the associated strong blowing would prevent the needed transfer of heat from the atmosphere surrounding the droplet). As for the droplet Reynolds number, in regions where most of the spray vaporization occurs, the typical values of $\mathrm{Re}_{d}$ range from small compared with unity, for small droplet sizes, to values only moderately large compared with unity. For small $\mathrm{Re}_{d}$ the convective transport terms associated with the slip velocity are negligible in the gas-phase near-field region of the droplets, resulting in temperature and concentration fields with spherical symmetry. The convective transport due to slip flow becomes important in the non-spherico-symmetrical Oseen far-field region, scaled with $a / \operatorname{Re}_{d}$, which does not need to be described when evaluating the nearfield Stokes flow, because this matches directly with the first approximation given by the ambient field.

In the relevant distinguished limit $\operatorname{Re}_{d} \sim 1$, the molecular-transport effects on the near-droplet flow field, and also the vorticity, are confined to radial distances $r \sim a$ from the droplet and to the droplet wake, where the exchanges between the particle and the gas are collected and transported downstream with the local mean gas slip velocity. At downstream distances of order $l_{d}$ the width of the wake is of order $\sqrt{a l_{d}}$, and the relative variations of temperature and fuel mass fraction across the wake, of order unity at distances of order $a$, have been reduced by a factor $a / l_{d}$. Similarly, at these distances $\sim l_{d}$ the spatial variations of velocity in the wake are of order $\left|\mathbf{v}-\mathbf{v}_{d}\right| a / l_{d}$, whereas the azimuthal vorticity, of order $\left|\mathbf{v}-\mathbf{v}_{d}\right| / a$ in the vicinity of the droplet, is reduced by a factor $\left(a / l_{d}\right)^{3 / 2}$ in the wake.

The vorticity in the wake determines the local distribution of the gas velocity relative to the homogenized gas, with a momentum flux 
(towards the droplet) equal to the difference between the droplet drag $\mathbf{f}$ and the momentum added to the gas phase by vaporization of the liquid fuel at the droplet surface $\dot{m} \mathbf{v}_{d}$. This is clearly visible in the droplet source term on the right-hand side of (10). Correspondingly, since the fuel vapor generated at the droplet surface is convected to the trailing wake, the flux of the excess of fuel vapor across the wake equals the droplet vaporization rate $\dot{m}$, as seen in the homogenized gas-phase equations (8) and (9). Similarly, the flux of the defect of enthalpy in the droplet wake equals the rate of heat transferred to the droplet $\dot{m} L_{v}+\dot{q}_{d}$, corrected to account for the enthalpy of the fuel vapor added to the wake by vaporization $\dot{m} h_{\mathrm{F}_{s}}$, giving the contribution emerging in (11).

We now begin by summarizing the expressions of the exchange rates, for values of $\operatorname{Re}_{d}$ small compared with unity, which, surprisingly, due to strong geometric effects remain fairly accurate as the Reynolds number increases to $\operatorname{Re}_{d} \sim 1$, so that the associated description provides sufficient accuracy for many spray-combustion applications.

For values of $\mathrm{Re}_{d}$ much smaller than unity the flow is dominated by molecular transport, leading to well-known results, including the familiar Stokes formula

$\mathbf{f}=6 \pi \mu a\left(\mathbf{v}-\mathbf{v}_{d}\right)$

for the force acting on the droplet. This expression neglects the presence of the Stefan flow associated with vaporization, which gives a uniform radial mass flux equal to the droplet vaporization rate $\dot{m}$. Consideration of the Stefan flow due to gasification leads to quantitative modifications to Stokes law (20), including a correcting factor, a function of $\lambda$, fairly close to unity when $\lambda \sim 1$ [50].

In the limit of small $\operatorname{Re}_{d}$ associated with (20) the distributions of temperature and fuel mass fraction around the droplet are spherically symmetrical. The temperature distribution $\widetilde{T}$ is obtained as a function of the radial coordinate scaled with the droplet radius $\tilde{r}$ by integrating the steady form of the energy equation subject to the boundary values $T$ and $T_{d}$ in the surrounding atmosphere and on the droplet surface, respectively. The integration is simplified by incorporating the commonly used assumption of constant gas thermal conductivity, although analyses are available that do not make use of this approximation [51-53]. The resulting problem

$\frac{\mathrm{d}}{\mathrm{d} \tilde{r}}\left(\lambda \widetilde{T}-\tilde{r}^{2} \frac{\mathrm{d} \widetilde{T}}{\mathrm{~d} \tilde{r}}\right)=0 \begin{cases}\tilde{r}=1: & \widetilde{T}=T_{d} \\ \tilde{r} \rightarrow \infty & \widetilde{T}=T\end{cases}$

can be integrated to give $\widetilde{T}=T-\left(T-T_{d}\right)$ $\left(1-e^{-\lambda / \tilde{r}}\right) /\left(1-e^{-\lambda}\right)$, which can be used to compute the rate of heat transferred to the droplet by heat conduction from the gaseous atmosphere $\dot{q}_{g}=4 \pi a \kappa(\mathrm{d} \widetilde{T} / \mathrm{d} \widetilde{r})_{\tilde{r}=1}$, giving $\dot{q}_{g}=4 \pi \kappa a$
$\left(T-T_{d}\right) \lambda /\left(e^{\lambda}-1\right)$, where $\lambda /\left(e^{\lambda}-1\right)$ is the Nusselt number accounting for the Stefan flow. According to the energy balance at the droplet surface, the heat transferred from the surrounding gas is employed to heat up the interior of the droplet and to vaporize the liquid fuel at the surface according to $\dot{q}_{g}=\dot{q}_{d}+L_{v} \dot{m}$, which can be rewritten in the form

$4 \pi \kappa a\left(\frac{T-T_{d}}{e^{\lambda}-1}-\frac{L_{v}}{c_{p}}\right) \lambda=\dot{q}_{d}$

providing a relationship between $\lambda$ and $\dot{q}_{d}$. Similarly, the fuel-vapor distribution around the droplet

$\widetilde{Y}_{\mathrm{F}}=Y_{\mathrm{F}}+\left(Y_{\mathrm{F}, S}-Y_{\mathrm{F}}\right)\left(1-e^{-\operatorname{Le}_{\mathrm{F}} \lambda / \tilde{r}}\right) /\left(1-e^{-\operatorname{Le}_{\mathrm{F}} \lambda}\right)$

is obtained by integrating the corresponding convection-diffusion conservation equation with boundary conditions $\widetilde{Y}_{\mathrm{F}}=Y_{\mathrm{F}}$ as $\tilde{r} \rightarrow \infty$ and $\widetilde{Y}_{\mathrm{F}}=Y_{\mathrm{F}, S}$ at $\tilde{r}=1$, where $Y_{\mathrm{F}, S}$ is the fuel-vapor mass fraction at the droplet surface. Here, a binary description is adopted for the fuel-vapor diffusion velocity, with $D_{\mathrm{F}}$ representing the binary diffusion coefficient of the gaseous fuel and nitrogen -which is the dominant component of the gas mixture surrounding the droplet- and $\mathrm{Le}_{\mathrm{F}}=\kappa$ / $\left(\rho c_{p} D_{\mathrm{F}}\right)$ being the corresponding Lewis number. For a mono-component fuel droplet, for which the radial flux of fuel vapor is equal to $\dot{m}$, we can use the fuel-vapor distribution $\widetilde{Y}_{\mathrm{F}}(\tilde{r})$ to obtain the relationship

$\lambda=\frac{1}{\mathrm{Le}_{\mathrm{F}}} \ln \left(\frac{1-Y_{\mathrm{F}}}{1-Y_{\mathrm{F}, S}}\right)$.

To close the problem, we use again the condition of thermodynamic equilibrium at the droplet surface to compute the fuel surface mass fraction using the Clausius-Clapeyron relation for the partial pressure of the fuel vapor at the droplet surface

$\frac{M_{s}}{M_{\mathrm{F}}} Y_{\mathrm{F}, S}=\exp \left(\frac{L_{v}}{R_{\mathrm{F}} T_{B}}-\frac{L_{v}}{R_{\mathrm{F}} T_{d}}\right)$,

which has an Arrhenius-type dependence on $T_{d}$, with a constant heat of vaporization $L_{v}$ per unit mass if the pressure in the chamber is not close to the critical pressure. Here, $R_{\mathrm{F}}=R^{\circ} / M_{\mathrm{F}}$ is the fuel gas constant and $T_{B}$ is the boiling temperature of the fuel at the chamber pressure. The mean molecular mass of the gas at the droplet surface $M_{s}=\left(Y_{\mathrm{F}, S} / M_{\mathrm{F}}+\sum_{i \neq \mathrm{F}}^{N_{s}} Y_{i, s} / M_{i}\right)^{-1}$ depends on $Y_{\mathrm{F}, S}$ and also on the mass fractions of the other chemical species at the droplet surface $Y_{i, s}$. Their values can be obtained in terms of their corresponding ambient values $Y_{i}$ by integrating the convection-diffusion balance equations for each species to give $Y_{i, s}=Y_{i} \exp \left(-\lambda \mathrm{Le}_{i}\right)$, where the Lewis numbers $\mathrm{Le}_{i}$ are evaluated with the binary diffusion coefficients of the given species through nitrogen, whose mass fraction is determined in this approximation from the equation $\sum_{i=1}^{N_{s}} Y_{i}=1$. 
Equations (23) and (24), supplemented with the expressions $Y_{i, s}=Y_{i} \exp \left(-\lambda \mathrm{Le}_{i}\right)$ for the computation of $M_{s}$, determine the values of $\lambda$ and $Y_{\mathrm{F}, S}$ in terms of $T_{d}$ and of the ambient mass fractions. For given values of $a$ and $T$, the resulting value of $\lambda$ can be used in (22) to determine $\dot{q}_{d}$ and in (19) to compute the associated droplet vaporization rate $\dot{m}$. The computation is simplified if the expression $M_{\mathrm{F}} / M_{s}=Y_{\mathrm{F}, S}+(1-$ $\left.Y_{\mathrm{F}, S}\right) M_{\mathrm{F}} / M_{\mathrm{N}_{2}}$ is employed, an approximation that accounts for the large differences of the molecular masses of the fuel vapor and $\mathrm{N}_{2}$, while taking the molecular mass of all other species equal to that of nitrogen. In that case, equation (24) can be used to determine $Y_{\mathrm{F}, S}$ as a function of $T_{d}$, while (23) gives explicitly $\lambda$ in terms of $Y_{\mathrm{F}, S}$ and $Y_{\mathrm{F}}$.

The above expressions (22)-(24) involve a number of physicochemical properties that are different for different fuels, with representative values given below in Table 1 . For the three different liquid fuels considered, straightforward evaluations using these properties indicate that the latent heat of vaporization satisfies $L_{v} \gg R_{\mathrm{F}} T_{B}$, which implies that the mass fraction of fuel vapor at the droplet surface, given by the Clausius - Clapeyron equation (24), remains exponentially small until the droplet temperature reaches values close to the boiling temperature. For those applications in which the injection temperature $T_{I}$ is sufficiently below $T_{B}$, i.e., its value is such that $\left(T_{B}-T_{I}\right) / T_{B} \gg\left[L_{v} /\left(R_{\mathrm{F}} T_{B}\right)\right]^{-1}$, there exists necessarily an initial heat up period during which $Y_{\mathrm{F}, S} \ll 1$, so that $Y_{\mathrm{F}, S}$ can be neglected with respect to unity in (23). If the atmosphere surrounding the droplet contains fuel vapor, then during this initial period there exists condensation $(\lambda<0)$, as inferred by (23), while if $Y_{\mathrm{F}}=0$ then $0<\lambda \ll 1$. In this last case, the thermal power transferred from the gas is dedicated to increasing the temperature of the yet non-vaporizing droplet, with a rate that simplifies to $\dot{q}_{d}=4 \pi \kappa a\left(T-T_{d}\right)$, as follows from (22) when $\lambda \ll 1$. This heat-up period ends when the liquid temperature reaches values close to the boiling temperature, i.e., $\left(T_{B}-T_{d}\right) / T_{B} \sim\left[L_{v} /\left(R_{\mathrm{F}} T_{B}\right)\right]^{-1} \ll 1$, when vaporization starts with values of the fuel-vapor mass fraction of order unity at the droplet surface. In this vaporizing period the droplet temperature remains approximately constant, with a value $T_{d} \simeq T_{B}$, so that $\dot{q}_{d} \simeq 0$, while the vaporization rate reduces to the well-known Spalding expression $\frac{\dot{m}}{4 \pi \kappa a / c_{p}}=\lambda=\ln \left(1+\frac{c_{p}\left(T-T_{B}\right)}{L_{v}}\right)$

derived by computing $\lambda$ from (22) with $\dot{q}_{d}=0$. Clearly, the approximation $T_{d}=T_{B}$ used in (25) is inaccurate when the gas temperature in the environment surrounding the droplet is close to the boiling temperature, i.e., $\left(T-T_{B}\right) / T_{B} \sim$ $\left[L_{v} /\left(R_{\mathrm{F}} T_{B}\right)\right]^{-1} \ll 1$.

Using the expressions given above to evaluate f, $\dot{m}$, and $\dot{q}_{d}$ in the droplet-balance equations (14)-(16) provides estimates for the characteristic times of droplet vaporization, droplet acceleration, and droplet heating. For instance, using (19) to evaluate $\dot{m}$ in (15) yields

$$
-\frac{1}{a^{3}} \frac{\mathrm{d} a^{3}}{\mathrm{~d} t}=3 \frac{D_{T}}{a^{2}} \frac{\rho}{\rho_{l}} \lambda=\frac{\lambda}{t_{v}},
$$

which defines a vaporization time $t_{v}=\frac{1}{3}\left(a^{2} / D_{T}\right)$ $\left(\rho_{l} / \rho\right)$, where $D_{T}=\kappa /\left(\rho c_{p}\right)$ denotes the gas thermal diffusivity. On the other hand, using Stokes law (20) for the drag force in (14) provides

$\frac{\mathrm{d} \mathbf{v}_{d}}{\mathrm{~d} t}=\frac{9}{2} \frac{v}{a^{2}} \frac{\rho}{\rho_{l}}\left(\mathbf{v}-\mathbf{v}_{d}\right)=\frac{\mathbf{v}-\mathbf{v}_{d}}{t_{a}}$,

where $t_{a}=\frac{2}{9}\left(a^{2} / v\right)\left(\rho_{l} / \rho\right)$ is the droplet acceleration (or accommodation) time, the so-called Stokes time, which can be alternatively expressed in the form $t_{a}=2 t_{v} /(3 \mathrm{Pr})$ in terms of the Prandtl number $\operatorname{Pr}=v / D_{T}$. It is worth noting that, since $\operatorname{Pr} \simeq 0.7$ for air, the times $t_{v}$ and $t_{a}$ are practically identical. Similarly, the characteristic droplet heating time $t_{q}=\left(c_{l} / c_{p}\right) t_{v}$ involved in $\mathrm{d} T_{d} / \mathrm{d} t=$ $\left(T-T_{d}\right) / t_{q}$, obtained by using the simplified rate $\dot{q}_{d}=4 \pi \kappa a\left(T-T_{d}\right)$ in (16), also is of order of the droplet vaporization time $t_{v}$, because the specificheat ratio $c_{l} / c_{p}$ is always of order unity.

Equations (20) and (22)-(24) provide a simplified description of the exchange sources for the computational modeling of evaporating sprays. More involved expressions are needed for slip flow with $\mathrm{Re}_{d}$ larger than unity or when increased accuracy is sought by incorporating physical phenomena that are not considered in the above derivation [20] (see also $[54,55]$ for summaries and assessments of different models). For instance, unsteady effects resulting from droplet acceleration can be accounted for, leading to corrections to the drag force and vaporization rate that have been computed by asymptotic methods [56].

As previously mentioned, effects of near-droplet convection associated with the slip velocity

Table 1

Values of physicochemical properties relevant for droplet heating and vaporization.

\begin{tabular}{llllllll}
\hline & $\left.M_{\mathrm{F}}[\mathrm{g} / \mathrm{mol})\right]$ & $\rho_{l}\left[\mathrm{~kg} / \mathrm{m}^{3}\right]$ & $c_{l}[\mathrm{~kJ} / \mathrm{kg}]$ & $L_{v}[\mathrm{~kJ} / \mathrm{kg}]$ & $T_{B}[\mathrm{~K}]$ & $L_{\mathrm{F}}$ & $L_{v} /\left(R_{\mathrm{F}} T_{B}\right)$ \\
\hline Heptane & 100 & 679.5 & 2.250 & $0.34 \times 10^{3}$ & 371 & 2.6 & 11.02 \\
Dodecane & 170 & 750 & 2.212 & $0.36 \times 10^{3}$ & 489 & 3.6 & 15.05 \\
Methanol & 32 & 791 & 2.484 & $1.1 \times 10^{3}$ & 337 & 1.2 & 12.56 \\
\hline
\end{tabular}


$\mathbf{v}-\mathbf{v}_{d}$ introduce corrections to the exchange rates that, surprisingly, remain moderately small as the Reynolds number increases, so that (20) and (22) (24) provide sufficient accuracy for $\operatorname{Re}_{d} \sim 1$. For instance, although the expression given in (20) tends to underpredict the drag force on the droplet as the Reynolds number increases, the underpredictions are not severe, i.e., the relative errors are of the order of $12 \%$ for $\operatorname{Re}_{d}=1$, increasing to $40 \%$ for $\mathrm{Re}_{d}=5$ [57].

As a final comment, it should be mentioned that the flow around the droplet is modified for droplets burning individually in an oxidizing atmosphere with a surrounding flame. For droplets moving relative to the ambient gas with a Reynolds number $\mathrm{Re}_{d}$ of order unity, the envelope diffusion flame around the droplet extinguishes when the droplet radius decreases below a critical value of the order of the thickness $\delta_{L}$ of the stoichiometric gaseous planar deflagration. For lower values of the droplet radius, the fuel vapor generated by the droplet will only burn in its wake, in a distributed manner. Conversely, a droplet may burn individually with a surrounding flame when its radius is larger than the critical extinction value, that being the case observed in the spraydeflagration experiments reported in [58], which consider large droplets with diameters exceeding 300 microns. When the droplet is surrounded by a flame, the expressions derived above for $\mathrm{Re}_{d} \ll 1$ should be modified. For instance, in the Spalding vaporization rate (25) the enthalpy difference $c_{p}\left(T-T_{B}\right)$ must incorporate an augmented effective ambient temperature that accounts for the presence of the flame. In the limit of infinitely fast reaction with the reasonable approximation of unity Lewis number adopted for $\mathrm{O}_{2}$, the associated enthalpy increase can be shown to be equal to the ambient oxygen mass fraction $Y_{\mathrm{O}_{2}}$ times the amount of heat released per unit mass of oxygen consumed in the chemical reaction $[45,59]$. The surrounding flame has been shown to affect also the force acting on the droplet [52]. Modifications would also be needed in the gas-phase equations, with the burning droplets appearing there as sources of heat and combustion products and as sinks for oxygen [53].

\section{Spray-combustion phenomenology}

The fuel vapor generated by the vaporizing droplets mixes with the surrounding hot air, enabling ignition to occur when a favorable equivalence ratio is encountered. Fuel sprays can be ignited using external sources such as electric sparks, torches or plasma jets, as is needed during the start and relight of jet engines and in the operation of gasoline direct-injection engines $[60,61]$. Forced ignition is not needed during the normal steady operation of continuous-combustion systems, such as that depicted in Fig. 2, which represents the typical arrangement found in gas turbines or industrial furnaces [60]. The manner in which combustion is stabilized downstream from the initial vaporization region is fundamentally dependent on the injection conditions. When the existing flow velocity is sufficiently low, combustion is established through partially premixed fronts that propagate along mixing layers in the nonuniform mixture created upstream by the vaporizing spray $[62,63]$. In many systems, however, the injection velocities are much higher than the characteristic deflagration speed, thereby precluding upstream triple-flame propagation. In that case, combustion stabilization must rely instead on the autoignition of the fuel-air mixture, which is facilitated by the high temperature of the surrounding gas, with ignition often occurring near the edge of the spray jet, where the temperatures are higher.

The resulting ignition dynamics depends on the dispersion of the droplets in the presence of turbulent motion [64]. Optimal droplet dispersion is achieved for values of the droplet Stokes time, defined in (27), of the order of the integral time scale of the large vortices in the mixing layer $[17,34,65,66]$, under which conditions droplets may cross the mixing layer at vortex-braid regions to vaporize on the other side surrounded by hot air. On the other hand, droplets with sufficiently small Stokes times behave as flow tracers and become entrained in the large-scale turbulent eddies, where they come into contact with the high-temperature air. The lower strain rates found in these near-core regions facilitate ignition, whereas the larger strain rates found in the vortex-braid regions prevent ignition from occurring there by limiting fuel residence times.

As suggested earlier for purely gaseous ignition [67], the unsteady unstrained flamelet -and also the closely related problem of the laminar coflow mixing layer- may provide an adequate representation of the spray ignition dynamics occurring at the low-strain interfaces wrapped around the vortices. Numerical and asymptotic analyses of group ignition of heptane and methanol spray streams by coflowing hot air were attempted recently [64], with a simple one-step Arrhenius model adopted for the chemistry description. The two main controlling parameters were seen to be the liquid mass-loading ratio $\alpha$ of the spray and the ratio of the droplet vaporization time $t_{v}$ to the characteristic chemical time for ignition $t_{c h}$, evaluated at the air-side temperature, both assumed to be of order unity in the integrations. The solution was seen to depend strongly on the thermochemical properties of the selected fuel. Thus, because of its smaller latent heat of vaporization $L_{v}$, heptane droplets vaporize faster than methanol droplets. As a result, as the mixing layer develops, heptane vapor becomes available for 
reaction earlier than methanol vapor, thereby leading to smaller ignition distances, a result in agreement with the ignition trends observed in previous numerical computations of ignition times in uniform spray mixtures [68]. Besides, the ignition of heptane is facilitated by its chemical heat release being more than twice that of methanol, resulting in a larger temperature increase per unit mass of fuel burnt that facilitates the self-acceleration of the chemical reaction rate, enabling a thermal runaway to take place. By way of contrast, the ignition of methanol proceeds gradually, in the form of a lean premixed flame that propagates slowly across the mixing layer from the hot air side. As shown in [64], the morphology of the ignition region is very sensitive to the specific values of the parameters selected. For instance, when air is employed as spray carrier, two-flame structures [69-73] are seen to emerge when the thermochemical properties of heptane are considered, but they are not observed for methanol.

As mentioned earlier, for all liquid fuels the mass of air needed to burn the unit mass of fuel, $S$, is a very large quantity (i.e., $S \simeq 15.2$ for heptane). As a result, in many applications, the partially premixed front originating at the ignition kernel burns completely the air that has been entrained upstream, while consuming only a limited fraction of the fuel available in the jet spray. The remaining gaseous fuel and accompanying fuel droplets burn downstream, in a diffusion flame that envelops the oxygen-starved spray jet. The resulting group-combustion configuration was envisioned as the predominant combustion regime in early theoretical analyses of dropletcloud combustion, to be discussed in Section 6 . The extent to which the partially premixed region contributes to the overall combustion process depends on the specific configuration and may also change depending on the operating conditions, as observed in direct numerical simulations [73], which also reinforce the qualitative description given above.

Figure 2 depicts an external diffusion-flame configuration, often encountered in applications, in which the diffusion flame stands off the droplet cloud, burning the ambient oxygen with the fuel that originates from the vaporizing droplets. In many applications, the gas-phase chemical reactions are fast, in that the characteristic time for fuel oxidation is much shorter than both the characteristic fluid-mechanical time and the dropletvaporization time. Under those conditions, the flame appears as a sheet, $\Sigma_{f}$, separating an internal oxygen-free region $\Omega_{\mathrm{F}}$ from an external region $\Omega_{\mathrm{O}}$ where no gaseous fuel is present in significant amounts. The associated Burke-Schumann limit of infinitely fast combustion will be discussed in Section 5 .

For the high-Reynolds-number flows typically encountered in liquid-fueled burners, the diffusion flame is embedded in thin mixing layers bounding the spray-carrier stream. These mixing layers are distorted and strained by the turbulent flow. A canonical problem that helps to investigate effects of strain on spray diffusion flames is the counterflow configuration [74], which will be addressed in Section 7. Sufficiently large values of the strain rate may lead to flame extinction [75]. When this occurs, the flame surface develops an incipient hole with a bounding edge flame that propagates along the mixing layer. The dynamics of these edge flames under the action of the external strain determines whether the flame hole reheals, through a reignition triple flame, or whether the extinction hole continues to increase, as the edge flame further retreats. While there exists substantial knowledge of many aspects of edge flames and triple flames in gaseous combustion [76,77], the associated spray problem has only been considered recently [78] and more work is needed to both clarify the structure of spray edge flames and quantify the dependences of their propagation velocity.

Large droplets with sufficient inertia may cross the mixing layers and move into the oxidizer stream, where they can possibly burn or vaporize individually or in small groups if favorable conditions are found. In principle, a closed diffusion flame may appear surrounding each individual droplet if their radius is large enough and favorable conditions for ignition are found as the droplet enters the oxidizer region [79]. The droplets in most practical combustion applications are however too small to sustain a flame in their vicinity. Therefore, as indicated in Fig. 2, many of the droplets that cross the spray diffusion flame can be expected to vaporize in $\Omega_{O}$ without a surrounding flame, generating fuel vapor that reacts with the existing oxygen in a distributed manner. Although the contribution of this distributed reaction to the total amount of heat released in the combustor can be expected to be negligible, these finite-rate effects are of utmost importance, since partial oxidation of the fuel vapor generated by the crossing droplets, especially in the cold regions found near the combustor walls, results in augmented emissions of $\mathrm{CO}$ and unburnt hydrocarbons. Extensions of the Burke-Schumann formulation using reduced mechanisms to describe finite-rate effects leading to pollutant emissions will be presented in Section 5 .

Before closing this overview, it is worth describing briefly the transient combustion phenomenology associated with the combustion cycle in diesel engines. Diesel-fuel injection into the cylinder begins shortly before top dead center. The spray starts to vaporize immediately as it mixes with the compressed hot air, creating a nonuniform mixture of fuel vapor and air, whose temperature continues to increase as a result of the homogeneous compression process that is occurring simultaneously, thereby enhancing the incipient chemical reaction. Autoignition occurs 
simultaneously at several near-stoichiometric hot spots in the bulk of the spray jet, where the most favorable conditions are met, leading to the formation of ignition fronts that sweep through the reactive mixture with a velocity of a few tens meters per second. This rapid process is accompanied by a significant increase of temperature and pressure in the cylinder that further accelerates the chemical reaction. The magnitude of these pressure and temperature increments depends on the fraction of the liquid fuel that was vaporized prior to ignition. As they propagate through the spray jet, the ignition fronts deplete the oxygen found in fuel-rich regions and the fuel vapor found in fuel-lean regions, leaving a diffusion flame at the instantaneous stoichiometric surface. As a result, an internal group combustion regime emerges following ignition, with the diffusion flame located within the spray, separating an inner oxygen-free region from an outer region free from gaseous fuel. In the subsequent evolution, the droplets found outside may burn individually or may vaporize, generating fuel vapor that mixes and reacts with the available air. As mentioned in Section 6, analyses of droplet-cloud combustion can be instrumental in investigating all these diesel-engine-combustion phenomena.

\section{The Burke-Schumann limit of infinitely-fast reaction in non-premixed spray flames}

In many liquid-fuel combustors the burning of the jet spray involves a thin nonpremixed flame surrounding the droplet cloud. The rates of the gasphase chemical reactions, which control the ignition distance and determine the structure of the partially premixed region, can be considered as infinitely fast for the diffusion flame that develops downstream; because the characteristic time for fuel oxidation is much shorter than both the characteristic fluid-mechanical time and droplet-vaporization times. Under those conditions, the nonpremixed spray flame is diffusion-controlled and can be consequently described in the first approximation by considering the limit of infinitely fast chemistry, to be addressed now following [80].

\subsection{The limit of infinitely fast reaction}

For simplicity, the presentation will consider that the chemical reaction between the oxygen of the air and the fuel vapor occurs according to the global irreversible step

$\mathrm{F}+s \mathrm{O}_{2} \rightarrow s_{\mathrm{CO}_{2}} \mathrm{CO}_{2}+s_{\mathrm{H}_{2} \mathrm{O}} \mathrm{H}_{2} \mathrm{O}+q$,

where $s, s_{\mathrm{CO}_{2}}$, and $s_{\mathrm{H}_{2} \mathrm{O}}$ are the mass of oxygen consumed and the mass of carbon dioxide and water vapor produced per unit mass of fuel burnt, so that $1+s=s_{\mathrm{CO}_{2}}+s_{\mathrm{H}_{2} \mathrm{O}}$, and $q$ is the corresponding amount of heat released. This overall reaction is an appropriate representation of the underlying chemistry when the concentrations of the intermediate reactive species are small compared with those of the main species appearing in (28), as it is typically the case in many combustion processes. Note that these intermediates, although appearing in small concentrations, determine the effective rate of the overall fuel-oxidation reaction (28), resulting from the interplay of elementary chemical reactions occurring at molecular level. This rate is necessary for describing premixed and partially premixed combustion, but is not essential for diffusion-controlled flames, as long as the strain conditions place the system away from extinction.

When the simplified description (28) is used to represent the overall combustion process, the conservation equations for reactants (9) reduce to

$$
\begin{aligned}
\frac{\partial}{\partial t} & \left(\rho Y_{\mathrm{F}}\right)+\nabla \cdot\left(\rho \mathbf{v} Y_{\mathrm{F}}\right)-\nabla \cdot\left(\frac{\rho D_{T}}{\mathrm{Le}_{\mathrm{F}}} \nabla Y_{\mathrm{F}}\right) \\
=-\omega_{\mathrm{F}} & +\sum_{j=1}^{N_{c}} n^{j} \dot{m}^{j}
\end{aligned}
$$

and

$$
\begin{aligned}
\frac{\partial}{\partial t}\left(\rho \widehat{Y}_{\mathrm{O}_{2}}\right)+ & \nabla \cdot\left(\rho \mathbf{v} \widehat{Y}_{\mathrm{O}_{2}}\right)-\nabla \cdot\left(\rho D_{T} \nabla \widehat{Y}_{\mathrm{O}_{2}}\right) \\
& =-S \omega_{\mathrm{F}} .
\end{aligned}
$$

where the oxygen mass fraction has been scaled with its value in the air stream to give the normalized variable $\widehat{Y}_{\mathrm{O}_{2}}=Y_{\mathrm{O}_{2}} / Y_{\mathrm{O}_{2,4}}$. Correspondingly, $S=s / Y_{\mathrm{O}_{24}}$ denotes the mass of air needed to burn the unit mass of fuel, a moderately large quantity, as previously mentioned. In the formulation, $\omega_{\mathrm{F}}$ represents the mass of fuel consumed per unit volume per unit time, which is related by $\omega_{\mathrm{F}}=-\dot{w}_{\mathrm{F}}$ to the production rate used above in (9). In writing Fick's law for the diffusion velocities, a unity Lewis number is assumed for $\mathrm{O}_{2}$, whereas a general non-unity Lewis number is used for the fuel, with representative values given in Table 1 .

To illustrate the Burke-Schumann limit of infinitely fast reaction rate, it is convenient to consider as an approximation the Arrhenius expression

$\omega_{\mathrm{F}}=\rho B \widehat{Y}_{\mathrm{O}_{2}} Y_{\mathrm{F}} \exp \left[-E_{a} /\left(R^{o} T\right)\right]$

for the reaction rate, including a frequency factor $B$ and an activation energy $E_{a}$, defining a characteristic temperature-dependent chemical time for fuel oxidation

$t_{c h}=B^{-1} \exp \left[E_{a} /\left(R^{o} T\right)\right]$.

Equation (31) can be used to express the reaction rates appearing in the conservation equations (29) and (30), giving for instance 
$\underbrace{\frac{1}{\rho} \mathcal{L}\left(Y_{\mathrm{F}}\right)}_{Y_{\mathrm{F}} / t_{p}}=-\underbrace{B \widehat{Y}_{\mathrm{O}_{2}} Y_{\mathrm{F}} \exp \left[-E_{a} /\left(R^{o} T\right)\right]}_{\widehat{Y}_{\mathrm{O}_{2}} Y_{\mathrm{F}} / t_{c h}}+\underbrace{n \dot{m} / \rho}_{1 / t_{p}}$

for the fuel balance, where $\mathcal{L}\left(Y_{\mathrm{F}}\right)$ represents the transport operator appearing on the left-hand side of (29). For the discussion, the anticipated orders of magnitude of the different competing phenomena have been indicated below each term, with the rates of accumulation and transport evaluated by assuming that the dominant fluid mechanical times are of the order of the droplet vaporization time and the source term evaluated for $\alpha \sim 1$.

Because of the large activation energy present in combustion reactions, the value of $t_{c h}$ given in (32) depends strongly on the local temperature, achieving its smallest value in the reaction region, where the temperature is of the order of the stoichiometric adiabatic flame temperature $T_{f}$. The Burke-Schumann limit arises in reactive sprays when the associated fuel oxidation time is much smaller than the characteristic vaporization and transport times, of order $t_{v}$, a condition often satisfied in practical burners whenever complete fuel consumption is to be achieved in the primary high-temperature combustion region. According to the above order-of-magnitude analysis, in this limit $t_{c h} \ll t_{v}$ of infinitely large Damköhler numbers the conservation equations for chemical species lead to the condition

$\widehat{Y}_{\mathrm{O}_{2}} Y_{\mathrm{F}}=0$,

indicating that the fuel and the oxidizer cannot coexist in the first approximation, except within a very thin reaction layer, to be described at leading order in the limit $t_{v} / t_{c h} \rightarrow \infty$ as an infinitesimally thin sheet, denoted by $\Sigma_{f}$ in Fig. 2. This flame sheet separates a region $\Omega_{\mathrm{F}}$, where $\widehat{Y}_{\mathrm{O}_{2}}=0$, from a region $\Omega_{\mathrm{O}}$, where $Y_{\mathrm{F}}=0$, whereas at the flame sheet both reactant mass fractions are simultaneously zero. In the solution that appears, the droplets lying in the oxygen-free region $\Omega_{\mathrm{F}}$ vaporize without chemical reaction, generating the fuel vapor that burns at the flame with the oxygen found in $\Omega_{\mathrm{O}}$.

\subsection{Gas-phase coupling functions for reactive sprays}

To remove the singular character of the solution associated with the limit $t_{v} / t_{c h} \rightarrow \infty$, when the reaction terms in the conservation equations become Dirac delta distributions along $\Sigma_{f}$, we follow the standard procedure indicated by Shvab and Zeldovich [81,82], consisting in eliminating the chemical reaction terms appearing in the conservation equations by appropriate linear combinations. The procedure is straightforward when the Lewis numbers of both reactants are unity, but it is somewhat more complicated when preferential diffusion is considered, because the linear combinations may result in coupling functions that are different for the diffusion terms and for the convective and accumulation terms [83,84]. For instance, when a non-unity Lewis number is present in (29), elimination of the chemical reaction by subtracting (30) from (29) times $S$ yields [80]

$$
\begin{aligned}
\frac{\partial}{\partial t}(\rho Z)+\nabla & \cdot(\rho \mathbf{v} Z)-\frac{S / \mathrm{Le}_{\mathrm{F}}+1}{S+1} \nabla \cdot\left(\rho D_{T} \nabla \widetilde{Z}\right) \\
= & \sum_{j=1}^{N_{c}} n^{j} \dot{m}^{j}
\end{aligned}
$$

involving two different mixture-fraction variables

$$
\begin{aligned}
& Z=\frac{S Y_{\mathrm{F}}-\widehat{Y}_{\mathrm{O}_{2}}+1}{S+1} \text { and } \\
& \widetilde{Z}=\frac{S Y_{\mathrm{F}} / \mathrm{Le}_{\mathrm{F}}-\widehat{Y}_{\mathrm{O}_{2}}+1}{S / \mathrm{Le}_{\mathrm{F}}+1} .
\end{aligned}
$$

A similar procedure must be utilized to eliminate the heat-release rate by chemical reaction $-\sum_{i=1}^{N_{s}} h_{i}^{o} \dot{w}_{i}=q \omega_{\mathrm{F}}$ from the energy equation. Since the Lewis number of oxygen is assumed to be unity, it is convenient to use (30) to derive the corresponding chemistry-free coupling function. The derivation is further facilitated by neglecting variations of the specific heat at constant pressure. Under those approximations, an appropriate linear combination of (12) and (30) with use made of (8) provides

$$
\begin{aligned}
\frac{\partial}{\partial t}(\rho H)+\nabla \cdot(\rho \mathbf{v} H)-\nabla \cdot\left(\rho D_{T} \nabla H\right) \\
=-\nabla \cdot \mathbf{q}_{R} \\
\quad-\sum_{j=1}^{N_{c}} n^{i}\left\{\dot{m}^{j}\left[q / S+L_{v}+c_{p}\left(T_{A}-T_{d}^{j}\right)\right]+\dot{q}_{d}^{j}\right\} \\
\quad+\frac{\mathrm{d} p_{o}}{\mathrm{~d} t}
\end{aligned}
$$

for the excess-enthalpy variable $H=c_{p}\left(T-T_{A}\right)+$ $\left(\widehat{Y}_{\mathrm{O}_{2}}-1\right) q / S$, where $T_{A}$ represents the temperature of the primary air-feed stream.

In the description of the limit of infinitely large Damköhler numbers, the three conservation equations for the reactants and energy are replaced with the chemistry-free equations (35) and (37), together with the condition (34) of non coexistence of the reactants. The flame is located where both the vapor fuel $Y_{\mathrm{F}}$ and the oxygen $\widehat{Y}_{\mathrm{O}_{2}}$ are simultaneously zero, corresponding to values of the mixture fraction $Z=Z_{s t}=1 /(1+S)$ and $\widetilde{Z}=\widetilde{Z}_{s t}=1 /\left(1+S /\right.$ Le $\left._{\mathrm{F}}\right)$. For $Z \geqslant Z_{s t}$ we find $\widehat{Y}_{\mathrm{O}_{2}}=0$, so that 
$Y_{\mathrm{F}}=\frac{Z-Z_{s t}}{1-Z_{s t}}=\frac{\widetilde{Z}-\widetilde{Z}_{s t}}{1-\widetilde{Z}_{s t}} \quad$ and

$c_{p}\left(T-T_{A}\right)=H+\frac{q}{S}$,

whereas $Y_{\mathbf{F}}=0$ for $Z \leqslant Z_{s t}$, giving

$\widehat{Y}_{\mathrm{O}_{2}}=1-\frac{Z}{Z_{s t}}=1-\frac{\widetilde{Z}}{\widetilde{Z}_{s t}}$ and

$c_{p}\left(T-T_{A}\right)=H+\frac{q}{S} \frac{Z}{Z_{s t}}$

These relationships link the values of $Z, \widetilde{Z}$, and $H$ and provide the mass fractions of reactants and the temperature in terms of the coupling functions everywhere in the flow field. If needed, source-free conservation equations that determine the product concentrations can be obtained from linear combinations accounting for non-unity Lewis numbers of $\mathrm{CO}_{2}$ and $\mathrm{H}_{2} \mathrm{O}$ [80].

\subsection{Distributed air-side fuel oxidation}

The formulation given above allows for the presence of droplets in $\Omega_{0}$. These droplets may be initially in $\Omega_{\mathrm{F}}$ and cross the flame at a given instant as a result of dispersion by turbulent eddies, or they may be already in $\Omega_{O}$ when the diffusion flame forms, as occurs in diesel engines with the droplets located in the outer part of the droplet cloud at ignition. When this arises, individual combustion of large droplets is then possible, provided that the droplets are sufficiently separated for the inter-droplet environment to host individual droplet flames and their Reynolds number $\mathrm{Re}_{d}$ does not exceed a critical extinction value. The Burke-Schumann formulation remains valid in the presence of droplets burning individually in $\Omega_{O}$, provided that the expression for the vaporization rate $\dot{m}$ is modified for the burning droplets, as indicated above at the end of Section 3.2.

It can be expected, however, that for the conditions found in many practical liquid-fueled burners, with typical droplet radii on the order of a few tens of microns, the droplets cannot burn individually with a surrounding flame because the fuel-oxidation time $t_{c h}$, although much smaller than the droplet vaporization time, is much larger than the characteristic diffusion time around the droplet. If $t_{c h}$ is also much larger than the characteristic interdroplet diffusion time $l_{d}^{2} / D_{T}$, so that the inequalities $l_{d}^{2} / D_{T} \ll t_{c h} \ll t_{v}$ hold, the fuel generated by droplets vaporizing in $\Omega_{\mathrm{O}}$ is homogeneously consumed in a distributed reaction occurring in the gas phase between droplets that can be described with the multi-continua formulation. According to the anticipated orders of magnitude displayed in (33), the fuel-vapor mass fraction in
$\Omega_{\mathrm{O}}$, of order $Y_{\mathrm{F}} \sim t_{c h} / t_{v} \ll 1$, can be determined with negligible transport effects. Equating the fuel-consumption rate by chemical reaction and the fuel-generation rate by droplet vaporization yields in the first approximation, from (25) and (31),

$$
\begin{aligned}
Y_{\mathrm{F}}= & \frac{4 \pi}{B} \frac{a D_{T} n}{\widehat{Y}_{\mathrm{O}_{2}}} \exp \left[E_{a} /\left(R^{o} T\right)\right] \\
& \times \ln \left[1+\frac{c_{p}\left(T-T_{B}\right)}{L_{v}}\right]
\end{aligned}
$$

as an explicit expression for the distribution of the fuel mass fraction, where the values of $a, D_{T}, n$, $\widehat{Y}_{\mathrm{O}_{2}}$, and $T$ are to be evaluated from the leadingorder Burke-Schumann solution. The accuracy of the above prediction is subject to the condition that the chemistry remains sufficiently fast for the transport rate to be negligible in (33). Because of the temperature dependence of the reaction rate, in regions of lower temperature the chemical reaction becomes slower. The associated fuel-consumption time (32) becomes comparable to the vaporization time when the temperature decreases to values of the order of a critical crossover temperature, $T_{c c}$, such that

$B^{-1} \exp \left(\frac{E_{a}}{R^{o} T_{c c}}\right)=t_{v}$.

In regions away from the flame where $T \sim T_{c c}$, the local Damköhler number

$\frac{t_{v}}{t_{c h}}=\exp \left(\frac{E_{a}}{R^{\circ} T_{c c}}-\frac{E_{a}}{R^{o} T}\right)$,

defined with use made of (32) and (41), becomes of order unity, so that transport effects enter to modify the analytic prediction (40), with larger corrections expected to appear at increasing distances from the flame. If the nondimensional activation energy $E_{a} /\left(R^{\circ} T_{c c}\right)$ is large, then (42) indicates that fuel consumption freezes as soon as the temperature falls below $T_{c c}$ (i.e., the local Damköhler number becomes exponentially small), whereas in regions where $T>T_{c c}$ fuel consumption is very rapid, giving the quasi-steady distribution (40) with exponentially small values of $Y_{\mathrm{F}}$. For $T<T_{c c}$, the fuel mass fraction is determined by a balance between transport and vaporization. Consideration of fuel transport in these cold regions would be needed in particular to determine emissions of unburnt hydrocarbons in liquid-fueled combustors. The computation of the small fuel-mass fraction as a higher-order correction to the Burke-Schumann solution would require integration of (29) in the region $Z<Z_{s t}$. The resulting problem is linear in $Y_{\mathrm{F}}$, because all of the remaining variables appearing in (29), including in particular $\widehat{Y}_{\mathrm{O}_{2}}$ and $T$, are to be evaluated from the leading-order solution. 


\subsection{Quantification of $\mathrm{CO}$ emissions}

The previous finite-rate results can be extended to account for more realistic fuel-oxidation descriptions. Although the detailed chemistry of liquid fuels typically involves a few thousand elementary reactions between a few hundred reactive species, flame studies have demonstrated that most of the chemical intermediates are in steady state, so that reduced chemical-kinetic descriptions with just a few overall steps provide sufficient accuracy $[85,86]$. For most purposes, combustion of a given hydrocarbon $\mathrm{C}_{\mathcal{M}} \mathrm{H}_{\mathcal{N}}$ can be described by introducing steady-state approximations for all but three chemical intermediates, namely, $\mathrm{CO}, \mathrm{H}_{2}$, and a radical representing the hydrogen-oxygen radical pool. For the lean conditions found in $\Omega_{\mathrm{O}}$, hydroxyl radicals are more abundant than $\mathrm{H}$ atoms [87], so it is best to develop the chemistry reduction with $\mathrm{CO}$, $\mathrm{H}_{2}$, and $\mathrm{OH}$ as species out of steady state, leading to the four overall reactions

$$
\begin{aligned}
& \mathrm{C}_{\mathcal{M}} \mathrm{H}_{\mathcal{N}}+\mathcal{R O H} \stackrel{\mathrm{I}}{\rightarrow} \mathcal{M C O}+(\mathcal{R}-\mathcal{M}) \mathrm{H}_{2} \mathrm{O} \\
& \quad+[\mathcal{M}+(\mathcal{N}-\mathcal{R}) / 2] \mathrm{H}_{2}, \\
& \mathrm{H}_{2}+\mathrm{O}_{2} \stackrel{\text { II }}{\rightleftharpoons} 2 \mathrm{OH}, \\
& 2 \mathrm{OH}+\mathrm{H}_{2} \stackrel{\text { III }}{\longrightarrow} \mathrm{H}_{2} \mathrm{O} \\
& \mathrm{CO}+\mathrm{H}_{2} \mathrm{O} \stackrel{\text { IV }}{\rightleftharpoons} \mathrm{CO}_{2}+\mathrm{H}_{2},
\end{aligned}
$$

representing fuel consumption, chain branching, chain recombination, and $\mathrm{CO}$ oxidation, with $\mathcal{R}$ denoting the number of radicals consumed per fuel molecule consumed. The value of the integer $\mathcal{R}, 1 \leqslant \mathcal{R} \leqslant \mathcal{N}$, depends on the details of the fuel-radical steady states. The corresponding overall rates (mols per unit volume per unit time) can be expressed as

$$
\begin{aligned}
& w_{\mathrm{I}}=k_{6 f} C_{\mathrm{F}} C_{\mathrm{OH}}+k_{7 f} C_{\mathrm{F}} C_{\mathrm{H}} \\
& w_{\mathrm{II}}=k_{1 f} C_{\mathrm{O}_{2}} C_{\mathrm{H}}-k_{1 b} C_{\mathrm{O}} C_{\mathrm{OH}} \\
& w_{\mathrm{III}}=k_{4 f} C_{\mathrm{M}} C_{\mathrm{O}_{2}} C_{\mathrm{H}} \\
& w_{\mathrm{IV}}=k_{5 f} C_{\mathrm{CO}} C_{\mathrm{OH}}-k_{5 b} C_{\mathrm{CO}_{2}} C_{\mathrm{H}}
\end{aligned}
$$

in terms of those of elementary reactions $\mathrm{H}+\mathrm{O}_{2}$

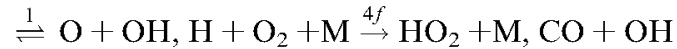
$\stackrel{5}{\rightleftharpoons} \mathrm{CO}_{2}+\mathrm{H}, \mathrm{C}_{\mathcal{M}} \mathrm{H}_{\mathcal{N}}+\mathrm{OH} \stackrel{6 f}{\rightarrow} \mathrm{C}_{\mathcal{M}} \mathrm{H}_{\mathcal{N}-1}+\mathrm{H}_{2} \mathrm{O}$ and $\mathrm{C}_{\mathcal{M}} \mathrm{H}_{\mathcal{N}}+\mathrm{H} \stackrel{7 f}{\rightarrow} \mathrm{C}_{\mathcal{M}} \mathrm{H}_{\mathcal{N}-1}+\mathrm{H}_{2}$, with $k_{j f}$ and $k_{j b}$ denoting the forward and backward reactionrate constants for each reaction $j$.

The concentrations of $\mathrm{H}$ and $\mathrm{O}$, needed in evaluating the overall reaction rates given in (44), can be obtained by assuming partial equilibrium of the fast shuffle reactions $\mathrm{H}_{2}+\mathrm{OH} \stackrel{2}{\rightleftharpoons} \mathrm{H}_{2} \mathrm{O}+\mathrm{H}$ and $\mathrm{OH}+\mathrm{OH} \stackrel{3}{\rightleftharpoons} \mathrm{H}_{2} \mathrm{O}+\mathrm{O}$ to give $\mathrm{C}_{\mathrm{H}}=K_{2} C_{\mathrm{H}_{2}} C_{\mathrm{OH}} /$ $C_{\mathrm{H}_{2} \mathrm{O}}$ and $C_{\mathrm{O}}=K_{3} C_{\mathrm{OH}}^{2} / C_{\mathrm{H}_{2} \mathrm{O}}$, where $K_{2}=k_{2 f} / k_{2 b}$ and $K_{3}=k_{3 f} / k_{3 b}$ are equilibrium constants. For this reduced description, conservation equations can be written for the fuel and for the different intermediates according to

$$
\begin{aligned}
& \mathcal{L}\left(Y_{\mathrm{F}}\right)=-M_{\mathrm{F}} w_{\mathrm{I}}+n \dot{m}, \\
& \mathcal{L}\left(Y_{\mathrm{CO}}\right)=M_{\mathrm{CO}}\left(\mathcal{M} w_{\mathrm{I}}-w_{\mathrm{IV}}\right), \\
& \mathcal{L}\left(Y_{\mathrm{H}_{2}}\right)=M_{\mathrm{H}_{2}}\left\{[\mathcal{M}+(\mathcal{N}-\mathcal{R}) / 2] w_{\mathrm{I}}-w_{\mathrm{II}}-w_{\mathrm{III}}+w_{\mathrm{IV}}\right\}, \\
& \mathcal{L}\left(Y_{\mathrm{OH}}\right)=M_{\mathrm{OH}}\left(-\mathcal{R} w_{\mathrm{I}}+2 w_{\mathrm{II}}-2 w_{\mathrm{II}}\right) .
\end{aligned}
$$

As in (33), the symbol $\mathcal{L}$ is used here to represent the transport differential operator for each chemical species.

In near-flame zones of $\Omega_{0}$ where the temperature is of the order of adiabatic flame temperature $T_{f}$, the chemical times associated with the four overall reactions are sufficiently small for the condition $t_{c h} \ll t_{v}$ to hold, so that the transport terms can be neglected in the first approximation in the above equations, reducing the computation of the concentrations of $\mathrm{C}_{\mathcal{M}} \mathrm{H}_{\mathcal{N}}, \mathrm{CO}, \mathrm{H}_{2}$, and $\mathrm{OH}$ to the solution of the reduced fuel conservation equation

$M_{\mathrm{F}} w_{\mathrm{I}}=n \dot{m}$,

indicating that fuel produced by droplet vaporization is immediately consumed by chemical reaction, along with the three steady-state expressions for the intermediates, which can be solved to give

$$
\begin{aligned}
C_{\mathrm{H}_{2}} & =\frac{k_{5 f}}{K_{2}\left(\gamma_{5 b}+A_{\mathrm{F}}\right) k_{4 f} C_{\mathrm{M}}} \frac{C_{\mathrm{H}_{2} \mathrm{O}} C_{\mathrm{CO}}}{C_{\mathrm{O}_{2}}} \\
C_{\mathrm{OH}} & =\left(\frac{k_{1 f}}{k_{4 f} C_{\mathrm{M}}}-B_{\mathrm{F}}\right)^{1 / 2}\left(\frac{k_{5 f}}{K_{3}\left(\gamma_{5 b}+A_{\mathrm{F}}\right) k_{1 b}}\right)^{1 / 2} C_{\mathrm{H}_{2} \mathrm{O}}^{1 / 2} C_{\mathrm{CO}}^{1 / 2}
\end{aligned}
$$

in terms of the reaction-rate ratio $\gamma_{5 b}=k_{5 b} C_{\mathrm{CO}_{2}} /\left(k_{4 f} C_{\mathrm{M}} C_{\mathrm{O}_{2}}\right)$ and the fuel-specific constants

$$
\begin{aligned}
& A_{\mathrm{F}}=\frac{\mathcal{M}}{\mathcal{M}+\mathcal{N}-2 \mathcal{R}} \text { and } \\
& B_{\mathrm{F}}=\frac{4 \mathcal{M}+\mathcal{N}}{4 \mathcal{M}+\mathcal{N}-2 \mathcal{R}} .
\end{aligned}
$$

Note that, according to (47), this steadystate description gives a zero $\mathrm{OH}$ concentration as the temperature decreases to a crossover value $T_{c c}$, defined in this case by the condition $k_{1 f}=B_{\mathrm{F}} k_{4 f} C_{\mathrm{M}}$. For $T<T_{c c}$, the expression (47) should be replaced by the condition $C_{\mathrm{OH}}=0$. The corresponding $\mathrm{CO}$-oxidation rate,

$w_{\mathrm{IV}}=\frac{A_{\mathrm{F}}}{\left(k_{1 b} K_{3}\right)^{1 / 2}}\left(\frac{k_{5 b}}{\gamma_{5 b}+A_{\mathrm{F}}}\right)^{3 / 2}\left(\frac{k_{1 f}}{k_{4 f} C_{\mathrm{M}}}-B_{\mathrm{F}}\right)^{1 / 2} C_{\mathrm{H}_{2} \mathrm{O}}^{1 / 2} C_{\mathrm{CO}}^{3 / 2}$,

obtained from (44) with use made of (47), also includes the crossover factor, and therefore vanishes at $T=T_{c c}$.

The expression (49) can be used to evaluate explicitly the $\mathrm{CO}$-oxidation rate appearing in the conservation equation for $\mathrm{CO}$, 


$$
\begin{aligned}
\frac{\partial}{\partial t}\left(\rho Y_{\mathrm{CO}}\right)+ & \nabla \cdot\left(\rho \mathbf{v} Y_{\mathrm{CO}}\right)-\nabla \cdot\left(\frac{\rho D_{T}}{\mathrm{Le}_{\mathrm{CO}}} \nabla Y_{\mathrm{CO}}\right) \\
& =\frac{\mathcal{M} M_{\mathrm{CO}}}{M_{\mathrm{F}}} n \dot{m}-M_{\mathrm{CO}} w_{\mathrm{IV}}
\end{aligned}
$$

written here with use made of (45) to express $w_{\mathrm{I}}$. Since the activation energy of reaction $1 f$ is moderately large, at temperatures above crossover the rate $w_{\Gamma V}$ is fast, and the associated $\mathrm{CO}$-oxidation time is much smaller than the characteristic fluid-mechanical time. In these hot regions, the transport terms in (50) can be neglected in the first approximation, giving the balance $\mathcal{M n m}=M_{\mathrm{F}} w_{\mathrm{IV}}$ for the $\mathrm{CO}$ concentration in this region of large Damköhler numbers. The equation can be solved for $C_{\mathrm{CO}}$ to give

$$
\begin{aligned}
C_{\mathrm{CO}}= & \frac{\gamma_{5 b}+A_{\mathrm{F}}}{k_{5 b}}\left(\frac{k_{1 b} K_{3}}{M_{\mathrm{F}}^{2} C_{\mathrm{H}_{2} \mathrm{O}}}\right)^{1 / 3}\left(\frac{k_{1 f}}{k_{4 f} C_{\mathrm{M}}}-B_{\mathrm{F}}\right)^{-1 / 3} \\
& \times[(\mathcal{M}+\mathcal{N}-2 \mathcal{R}) n \dot{m}]^{2 / 3} .
\end{aligned}
$$

This transportless expression fails however as the temperature approaches crossover, because the rate (49) decreases to zero, with the result that (51) incorrectly predicts an infinite CO concentration at $T=T_{c c}$. Inclusion of $\mathrm{CO}$ transport readily avoids this singularity, imposes a limit $Y_{\mathrm{CO}} \sim\left(\mathcal{M} M_{\mathrm{CO}} n \dot{m} t_{v}\right) /\left(\rho M_{\mathrm{F}}\right)$ on $C_{\mathrm{CO}}$ in terms of the local value of $n \dot{m}$ (small in the region where freezing occurs), and enables the correct description of $\mathrm{CO}$ to be obtained for $T<T_{c c}$, where $w_{\mathrm{TV}}=0$ and the $\mathrm{CO}$ generated by partial oxidation of the vaporized fuel is transported away without significant oxidation to $\mathrm{CO}_{2}$. In integrating (50) in $\Omega_{O}$ one may use the concentrations of $\mathrm{O}_{2}, \mathrm{H}_{2} \mathrm{O}, \mathrm{CO}_{2}$, temperature, number of droplets per unit volume $n$, and radius $a$ corresponding to the leading-order Burke-Schumann solution to evaluate the vaporization rate $n \dot{m}$ and the $\mathrm{CO}$-oxidation rate from (25) and (49). Clearly, similar ideas could be employed to generate an equation for describing the generation of other pollutants, notably NO.

\section{Vaporization and combustion of fuel-spray clouds}

The description given in Section 2 illustrates the importance of group effects, with the mean gas-phase environment generated collectively by the droplets determining the overall vaporization and combustion dynamics of the spray. Elementary problems involving a limited number of competing phenomena serve to illustrate the interplay of the different physicochemical processes. Regimes of group vaporization and com- bustion of sprays have been defined by considering quasisteady solutions to uniform, spherically symmetric, quiescent fuel-droplet clouds [88-94]. Under appropriate simplifying assumptions, a single dimensionless parameter, called the group combustion number [88], has been reasoned to control the energy and mass transfer between the droplet cloud and the surrounding atmosphere. Since this number is widely used in applications, it is of interest to discuss its connection with the spray parameters and characteristic times identified above by addressing the problem of transient vaporization of a monodisperse droplet cloud in a hot air environment at constant pressure.

\subsection{The continuum, homogenized description of droplet-cloud vaporization}

Let us consider the temporal evolution of a spherical cloud of radius $R_{o}$ containing air and $N_{o}$ uniformly distributed droplets of radius $a_{o}$ and initial temperature $T_{I}$. An unbounded atmosphere of hot air with temperature $T_{A}>T_{I}$ and density $\rho_{A}$ surrounds the cloud. The initial number of droplets per unit volume in the cloud, $n_{o}=N_{o} /\left[(4 / 3) \pi R_{o}^{3}\right]$, defines the interdroplet distance $l_{d}=n_{o}^{-1 / 3}$, which is assumed to be in the range $R_{o} \gg l_{d} \gg a_{o}$, so that the condition (7) is satisfied. The droplet number density together with the droplet radius and the gas density defines the liquid mass-loading ratio, $\alpha=(4 \pi / 3) a_{o}^{3} n_{o} \rho_{l} / \rho_{A}$; when $\alpha \sim 1$ we can expect two-way coupling between the phases. The cloud evolution is mainly determined by the competition of droplet vaporization with heat conduction across the droplet cloud. The rate of the former is measured by the characteristic droplet vaporization time $t_{v}$, given in (26) as a function of the initial values of the droplet radius $a_{o}$ and air density and thermal diffusivity $\rho_{A}$ and $D_{T_{A}}$, whereas the heat conduction time based on the cloud radius $t_{c}=R_{o}^{2} / D_{T_{A}}$ characterizes gas-phase heat conduction from the surrounding atmosphere. Another significant parameter is $T_{A} / T_{B}$.

The description of the problem delineated above requires integration of (8), (9), and (12) for the gas phase together with (13)-(17) for the liquid phase, supplemented with the equation of state and with additional equations for the droplet source terms $\mathbf{f}, \dot{m}$, and $\dot{q}_{d}$. Since the heat needed to heat up and vaporize the equilibrium droplet cloud must come from the surrounding hot air, it is appropriate to use the heat conduction time $t_{c}$ to scale the problem. Introducing the dimensionless variables $\hat{t}=t / t_{c}, \quad \hat{r}=r / R_{o}, \quad \hat{v}=v_{r} /$ $\left(D_{T_{A}} / R_{o}\right), \quad \widehat{T}=T / T_{A}, \hat{\rho}=\rho / \rho_{A}, \hat{n}=n / n_{o}, \hat{a}=$ $a / a_{o}, \quad \widehat{T}_{d}=T_{d} / T_{A}, \quad$ and $\hat{v}_{d}=v_{d_{r}} /\left(D_{T_{A}} / R_{o}\right)$, reduces the conservation equations for the gas phase to 


$$
\begin{aligned}
& \frac{\partial \hat{\rho}}{\partial \hat{t}}+\frac{1}{\hat{r}^{2}} \frac{\partial}{\partial \hat{r}}\left(\hat{r}^{2} \hat{\rho} \hat{v}\right)=\alpha \frac{t_{c}}{t_{v}} \hat{n} \hat{a} \widehat{T}^{\sigma} \lambda, \\
& \frac{\partial \hat{\rho} Y_{\mathrm{F}}}{\partial \hat{t}}+\frac{1}{\hat{r}^{2}} \frac{\partial}{\partial \hat{r}}\left(\hat{r}^{2} \hat{\rho} \hat{v} Y_{\mathrm{F}}\right)-\frac{1}{\hat{r}^{2}} \frac{\partial}{\partial \hat{r}}\left(\frac{\hat{r}^{2} \widehat{T}^{\sigma}}{\mathrm{Le}_{\mathrm{F}}} \frac{\partial Y_{\mathrm{F}}}{\partial \hat{r}}\right) \\
& \quad=\alpha \frac{t_{c}}{t_{v}} \hat{n} \hat{a} \widehat{T}^{\sigma} \lambda, \\
& \frac{\partial \hat{\rho} \widehat{T}}{\partial \hat{t}}+\frac{1}{\hat{r}^{2}} \frac{\partial}{\partial \hat{r}}\left(\hat{r}^{2} \hat{\rho} \hat{v} \widehat{T}\right)-\frac{1}{\hat{r}^{2}} \frac{\partial}{\partial \hat{r}}\left(\hat{r}^{2} \widehat{T}^{\sigma} \frac{\partial \widehat{T}}{\partial \hat{r}}\right) \\
& \quad=-\alpha \frac{t_{c}}{t_{v}} \hat{n} \hat{a} \widehat{T}^{\sigma}\left[\left(\widehat{T}-\widehat{T}_{d}\right) \frac{\lambda}{e^{\lambda}-1}-\widehat{T}_{d} \lambda\right],
\end{aligned}
$$

to be supplemented with $\hat{\rho} \widehat{T}=\left[1-\left(1-M_{A}\right]\right.$ $\left.\left.M_{\mathrm{F}}\right) Y_{\mathrm{F}}\right]^{-1}$, where $M_{A} / M_{\mathrm{F}}$ denotes the air-to-fuel molecular-mass ratio. A simple power law with exponent $\sigma=0.7$ has been adopted for the temperature dependence of the transport coefficients. Radiative heat transfer has been neglected in writing (54), along with changes in specific heat. The source terms in (52)-(54) are proportional to $\hat{n}$, which satisfies the conservation law

$\frac{\partial \hat{n}}{\partial \hat{t}}+\frac{1}{\hat{r}^{2}} \frac{\partial}{\partial \hat{r}}\left(\hat{r}^{2} \hat{n} \hat{v}_{d}\right)=0$,

involving the droplet velocity $\hat{v}_{d}$. This parabolic system of equations must be complemented with the simultaneous Lagrangian description of the variation of the droplet properties $\hat{a}, \widehat{T}_{d}, \hat{v}_{d}$, given by

$$
\frac{\mathrm{d} \hat{a}^{3}}{\mathrm{~d} \hat{t}}=-\frac{t_{c}}{t_{v}} \hat{a} \widehat{T}^{\sigma} \lambda
$$

$\hat{a}^{3} \frac{\mathrm{d} \widehat{T}_{d}}{\mathrm{~d} \hat{t}}=\frac{t_{c}}{t_{v}} \frac{c_{p}}{c_{l}} \hat{a} \widehat{T}^{\sigma}\left[\left(\widehat{T}-\widehat{T}_{d}\right) \frac{\lambda}{e^{\lambda}-1}-\frac{L_{v}}{c_{p} T_{A}} \lambda\right]$,

$\hat{a}^{3} \frac{\mathrm{d} \hat{v}_{d}}{\mathrm{~d} \hat{t}}=\frac{3 \operatorname{Pr}}{2} \frac{t_{c}}{t_{v}} \hat{a} \widehat{T}^{\sigma}\left(\hat{v}-\hat{v}_{d}\right)$,

along the droplet paths $\hat{r}_{d}(\xi, \hat{t})$, given by the solution of $\mathrm{d} \hat{r}_{d} / \mathrm{d} \hat{t}=\hat{v}_{d}$ in terms of its initial radial location $\hat{r}_{d}=\xi$. The source terms in the above equations, which are proportional to $\dot{m}$ and $\dot{q}_{d}$, have been written using (19) and (22) in terms of $\lambda$, which is determined using (23), i.e., $\lambda=\mathrm{Le}_{\mathrm{F}}^{-1} \ln \left[\left(1-Y_{\mathrm{F}}\right) /\left(1-Y_{\mathrm{F}, S}\right)\right]$, as a function of $Y_{\mathrm{F}}$ and $Y_{\mathrm{F}_{s}}$. The latter is given by the ClausiusClapeyron relation (24) in terms of $\widehat{T}_{d}$, with $M_{\mathrm{F}} / M_{s}=Y_{\mathrm{F}_{s}}+\left(1-Y_{\mathrm{F}_{s}}\right) M_{\mathrm{F}} / M_{A}$, thereby completing the formulation.

Different possible sets of initial conditions can be considered [94]. For example, one may assume that the droplets are suddenly placed in the unperturbed hot-air environment at the start of the integration, with the uniform gas temperature $T_{A}$ everywhere at $t=0$. Perhaps a more realistic initial condition, especially in connection with controlled experiments in which the spray is formed upstream from the injection section, follows from assuming that the spray is initially in equilibrium, so that $\lambda=0$ and $T=T_{d}$ in the cloud, as is required for the right-hand side terms of (52)(54), (56), and (57) to vanish. The associated initial conditions for (52) (55) are $\hat{\rho}-\hat{\rho}_{I}=\widehat{T}-$ $\widehat{T}_{I}=Y_{\mathrm{F}}-Y_{\mathrm{F}_{I}}=\hat{n}-1=0$ for $\hat{r} \leqslant 1$ and by $\hat{\rho}-1=\widehat{T}-1=Y_{\mathrm{F}}=\hat{n}=0$ for $\hat{r}>1$, where $\hat{\rho}_{I}$ is the interdroplet gas density associated with $\widehat{T}=\widehat{T}_{I}$ and $Y_{\mathrm{F}_{l}}$, with $Y_{\mathrm{F}_{I}}$ determined from evaluating (24) at $T_{d}=T_{I}$. Correspondingly, the integration of (56)-(58) is initiated with $\hat{a}-1=$ $\hat{v}_{d}=\widehat{T}_{d}-\widehat{T}_{I}=0$. Boundary conditions for $\hat{t}>0$, needed to integrate (52)-(54) are given by $\hat{v}=\partial \widehat{T} / \partial \hat{r}=\partial Y_{\mathrm{F}} / \partial \hat{r}=0$ at $\hat{r}=0$ and $\widehat{T}-1=$ $Y_{\mathrm{F}}=0$ as $\hat{r} \rightarrow \infty$.

Many of the early numerical investigations of spherical droplet-cloud vaporization [88-94] neglected the motion of the droplets induced by the gas expansion along with the resulting nonuniformities in $\hat{n}$, both having in general a nonnegligible effect on the solution, as shown in $[95,96]$. These effects are effectively handled in the multicontinua formulation outlined above. It is worth mentioning that, although the droplets in the interior of the cloud may conceivably accelerate to overtake those located farther outside, with the result that droplets originating at different $\xi$ end up occupying the same radial location, such overtaking events were not observed in computations. If they occur, i.e., for initial conditions or parametric values different from those considered here, the associated duplicity should be taken into account when evaluating the droplet properties at a given location (e.g., by creating a new droplet class containing the droplets that have been overtaken, in a similar procedure as that employed in Section 7 for counterflow flames).

For numerical studies of vaporization and combustion of spray clouds in constant-volume or variable-volume chambers [95], of interest for diesel-engine applications, the term $\mathrm{d} p_{o} / \mathrm{d} t$ must be retained in writing (54) from (12), while the effect of the finite size of the combustion chamber could be effectively incorporated by introducing a moving or stagnant external wall at a finite radius $\hat{r}=R_{e} / R_{o}$. Since near stoichiometric conditions are of interest in diesel-engine combustion, values of $R_{e} / R_{o} \sim(\alpha S)^{1 / 3}$ should be considered in integrations. Such formulations could be employed to investigate autoignition by compression, providing the distributions of temperature and composition encountered immediately before ignition [97]. They would also be useful for studying the subsequent evolution of the diffusion flame generated upon ignition, which could be described in the limit of infinitely fast combustion.

\subsection{Parameters controlling droplet-cloud vaporization}

The solution depends on two fundamental independent parameters, the initial mass-loading 
ratio $\alpha$, which modulates the extent of interphase coupling, and the characteristic time ratio $t_{c} / t_{v}$, which measures the competition of heat transfer from the surrounding atmosphere with droplet vaporization. The product $\alpha t_{c} / t_{v}$, which measures the extent of spray vaporization in (52)-(54), is essentially the group combustion number $\mathrm{G}$ introduced by Chiu and co-workers [88], variants of which were also used by other early investigators [90,92-94]. Although $\alpha t_{c} / t_{v}$ can be expressed as $\alpha t_{c} / t_{v}=4 \pi n_{o} a_{o} R_{o}^{2}=3 N_{o} a_{o} / R_{o} \sim N_{o}^{2 / 3} a_{o} / l_{d}$,

involving either the initial droplet-number density $n_{o}$ or the total number of droplets in the cloud $N_{o}$, the physical interpretation of the resulting expressions is not transparent. Besides, accounting separately for the two times involved in the problem facilitates consideration of the effect of enhanced heat transfer by turbulent transport, of interest in realistic applications. For instance, to characterize approximately the rate of heat transfer one could employ the gas-phase effective heattransfer time $t_{c}^{*}=R_{o}^{2} / D_{t}$, defined by replacing the molecular diffusivity with a turbulent diffusivity $D_{t}$. Since exchanges between the two phases are still controlled by molecular transport in the immediate vicinity of the droplet, the resulting effective group combustion number $\alpha t_{c}^{*} / t_{v}$ would display a linear dependence on the ratio $D_{T_{A}} / D_{t}$, a function of the flow Reynolds number accounting approximately for the effect of turbulence.

The distinguished limit $t_{c} / t_{v}=O(1)$ and $\alpha=O(1)$ of droplet-cloud vaporization with two-way coupling between the liquid and gas phases requires numerical integration of the complete problem (52)-(58). Simplifications arise when the initial droplet temperature $T_{d}=T_{I}$ is close to the boiling temperature $T_{B}$, the case considered in the sample results of Fig. 3, for which $\widehat{T}_{d} \simeq \widehat{T}_{B}=T_{B} / T_{A}$ at all times, with the result that the evolution equation (57) is no longer needed. The expansion of the gas, which is initially very strong at the separating interface, is seen to induce significant droplet motion, as can be seen in the droplet trajectories shown in the bottom plot, which are extended in time until the droplet disappears. The slip velocity $\hat{v}-\hat{v}_{d}$, which is very large in the beginning, decays for $\hat{t} \sim 1$. Once the thermal wave reaches the center, vaporization occurs throughout the cloud, so that when the boundary droplet disappears for $\hat{t} \simeq 5.6$ the radius of the central droplet has decreased already to $\hat{a} \simeq 0.19$.

The limit of sheath vaporization [93] arises for $\alpha t_{c} / t_{v} \gg 1$, as can be seen from (52) and (53), which lead in this limit to the condition $\hat{a} \lambda=0$, indicating the existence of a thin vaporization front located at $\hat{r}=\hat{r}_{s}(\hat{t})$ separating and outer region for $\hat{r}>\hat{r}_{s}$ where no droplets are found and an inner region for $\hat{r}<\hat{r}_{s}$ where the vaporization rate is identically zero. Inside the cloud, where $\lambda=0$, the temperature must satisfy $\widehat{T}=\widehat{T}_{d}$, as follows from (54) in this limit. The vaporization front bounding the shrinking cloud moves slowly with a characteristic time that can be seen to be of order $\alpha t_{c}$, so that if $\alpha \gg 1$ the solution evolves in a quasi-steady fashion for $\hat{t}=t / t_{c} \gg 1$. The near-equilibrium stagnant solution in the cloud and the outer droplet-free, quasisteady solution for $\hat{r}>\hat{r}_{s}$ must satisfy appropriate jump conditions at the vaporization layer, providing an equation for $\mathrm{d} \hat{r}_{s} / \mathrm{d} \hat{t}$ that yields a prediction for the cloud life time, $\sim \alpha t_{c}$, upon integration (see the parallel analysis given in [98] for the cylindrical droplet cloud). Note that the sheath solution is somewhat more complex in the alternative limiting case $t_{c} / t_{v} \gg 1$ with $\alpha=O(1)$, corresponding also to large values of $\mathrm{G}=\alpha t_{c} / t_{v} \gg 1$, because in that case the cloud life time is of order $t_{c}$ and the solution outside the vaporization layer remains unsteady during the vaporization process.

\section{Counterflow spray flames}

A canonical problem of interest in connection with the high-Reynolds-number flows typically encountered in burners is that of the counterflow mixing layer. Counterflow structures that move
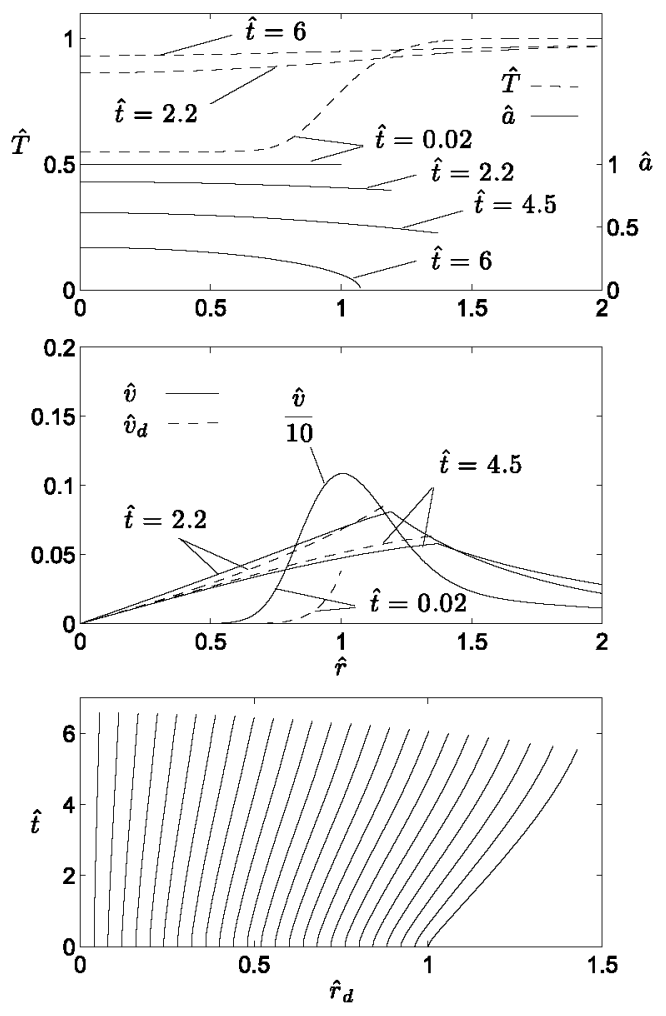

Fig. 3. The evolution of the vaporizing droplet cloud as obtained from integration of (52)-(58) for $\widehat{T}_{I}=\widehat{T}_{B}=$ $0.55, L_{v} /\left(c_{p} T_{A}\right)=0.48, \mathrm{Le}_{\mathrm{F}}=2.6, Y_{\mathrm{F}_{T}}=0, \alpha=1.0$, and $t_{c} / t_{v}=1$. The lower plot shows droplet trajectories, extended in time until the droplet disappears. 
with the mean velocity can be abstracted from the interface dynamics of shear and mixing layers [99]. Similarly, local counterflow configurations emerge in typical combustion chambers around the stagnation point that forms near the injector exit as a result of vortex breakdown of the swirling air-feed stream [100]. Counterflow configurations have been widely employed in laboratory experiments for studying reacting and non-reacting spray flows $[79,101-106]$ as well as in numerical simulations to address the effects of strain on spray flames $[75,104,107-116]$. The typical experimental arrangement involves two opposing nozzles of radius $R$ located at a distance $h$. The resulting coaxial counterflowing jets, one of air and the other containing an inert gas carrying the fuel spray, produce an axisymmetric laminar stagnation-point flow with gas velocity $\mathbf{v}=(u, v)$. The Reynolds number $\operatorname{Re}=U R / v_{s}$, based on the characteristic gas velocity $U$ at the nozzle exit and gas kinematic viscosity $v_{s}$ of the spraycarrier gas, is moderately large in typical applications, so that the flow of the counterflowing streams is nearly inviscid. Mixing between both streams occurs only in a thin layer located about the separating surface, whose characteristic thickness is $\delta_{m} \sim R / \operatorname{Re}^{1 / 2} \ll R$

The computation of the inviscid flow in the outer streams involves in principle integration of the Euler equations for the gas phase coupled to the solution for the liquid phase, with the outer jet boundaries and the interface separating the two jets appearing as free surfaces to be obtained as part of the computation. This nontrivial calculation provides, in particular, the structure of the inviscid gas flow near the stagnation point, where the radial and axial velocity components on the spray side of the mixing layer are found to increase away from the stagnation point according to

$v=A_{s} r / 2$ and $u=-A_{s} z$,

where $r$ and $z$ are the radial and axial coordinates measured from the apparent stagnation point and $A_{s}$ is the local strain rate on the spray side of the mixing layer. The value of $A_{s}$, a quantity of order $U / R$ that depends on the specific experimental setup (notably on the values of the inter-nozzle separation $h / R$ and of the inert-to-air density ratio $\rho_{s}\left(\rho_{A}\right)$, determines the structure of the thin mixing layer formed around the stagnation point, which exhibits a self-similar solution in terms of the distance $z$ to the stagnation plane. Note that the corresponding strain rate found on the air side is in general different, with a value $A_{A}=A_{s} \sqrt{\rho_{s} / \rho_{A}}$ dictated by the condition of negligible pressure variation across the mixing layer.

\subsection{Droplet dynamics in counterflow mixing layers}

The dynamics of droplets in counterflow mixing layers is known to depend fundamentally on the droplet Stokes number St [74,103,117-119], given by the ratio of the characteristic droplet accommodation time $t_{a}$, defined in (27), to the characteristic flow time. Using the reciprocal of the spray-side strain rate $A_{s}$ as a representative value of the latter yields

$\mathrm{St}=A_{s} t_{a}=\frac{2}{9} A_{s} \frac{a_{o}^{2}}{v_{s}} \frac{\rho_{l}}{\rho_{s}}$,

where $a_{o}$ is the initial droplet radius. For small values of $\mathrm{St}$, the droplets behave as flow tracers, and their trajectories follow closely those of the gas particles, while in the opposite limit, St $\gg 1$, the droplets move in nearly ballistic trajectories weakly influenced by aerodynamic forces. In the distinguished regime corresponding to values of St of order unity the inertia of the droplets plays an important role in their trajectories.

For the counterflow, there exists a critical value of the Stokes number $\mathrm{St}_{c}$ of order unity below which the droplets cannot cross the stagnation plane, but tend to accumulate there instead $[74,103,118,119]$. Conversely, droplets with Stokes numbers above the critical value cross the stagnation plane, following several oscillations before they vaporize completely $[75,120,121]$ (see also [74] for experimental visualizations of this phenomenon). It is evident that any strained flamelet model for spray combustion based on the counterflow needs to consider these effects.

The computation of the droplet trajectories $\mathbf{x}_{d}(t)=\left(r_{d}, z_{d}\right)$ and of the associated value of $\mathrm{St}_{c}$ requires integration of (13) and (14). In general, the calculation necessitates simultaneous determination of the evolution of the droplet radius and of the gas-phase velocity, needed to determine the force acting on the droplet. A well-known simple solution arises when droplet vaporization is neglected, along with changes of the gas velocity from the stagnation-point potential solution, which is given on both sides by (59) when $\rho_{s}=$ $\rho_{A}$. For a droplet released at $\mathbf{x}_{d}=\left(r_{I}, z_{I}\right)$ with initial injection velocity $\mathbf{v}_{d}=\left(v_{d}, u_{d}\right)=\left(r_{I} A_{s} / 2\right.$, $\left.-z_{I} A_{s}\right)$, equal to the gas velocity, the problem reduces to that of integrating the uncoupled second-order equations

$t_{a} \frac{\mathrm{d}^{2} r_{d}}{\mathrm{~d} t^{2}}+\frac{\mathrm{d} r_{d}}{\mathrm{~d} t}-\frac{1}{2} A_{s} r_{d}=0, t=0\left\{\begin{array}{l}r_{d}=r_{I} \\ \mathrm{~d} r_{d} / \mathrm{d} t=r_{I} A_{s} / 2\end{array}\right.$

and

$$
t_{a} \frac{\mathrm{d}^{2} z_{d}}{\mathrm{~d} t^{2}}+\frac{\mathrm{d} z_{d}}{\mathrm{~d} t}+A_{s} z_{d}=0, t=0\left\{\begin{array}{l}
z_{d}=z_{I} \\
\mathrm{~d} z_{d} / \mathrm{d} t=-z_{I} A_{s}
\end{array}\right.
$$

obtained from (13) and (14) with Stokes law (20) assumed for the droplet drag. Straightforward integration of (61) provides the evolution of the droplet radial location in terms of the dimensionless time $\tau=A_{s} t /(2 \mathrm{St})=t /\left(2 t_{a}\right)$,

$\frac{r_{d}}{r_{I}}=e^{-\tau}\left[\cosh (\tau \sqrt{2 \mathrm{St}+1})+\frac{1+\mathrm{St}}{\sqrt{4 \mathrm{St}-1}} \sinh (\tau \sqrt{2 \mathrm{St}+1})\right]$, 
which increases continuously regardless of the value of St. On the other hand, the character of the axial motion is fundamentally dependent on the value of St. For values of $\mathrm{St}<1 / 4$, the droplets approach the dividing streamsurface $z=0$ but never cross it, as can be seen by integrating (62) to give

$\frac{z_{d}}{z_{I}}=e^{-\tau}\left[\cosh (\tau \sqrt{1-4 \mathrm{St}})+\frac{1-2 \mathrm{St}}{\sqrt{1-4 \mathrm{St}}} \sinh (\tau \sqrt{1-4 \mathrm{St}})\right]$.

By way of contrast, oscillatory droplet motion of decreasing amplitude about the stagnation plane is observed for $\mathrm{St}>1 / 4$, with the droplets penetrating a distance of order $z_{I}$ into the opposing flow stream as described by

$\frac{z_{d}}{z_{I}}=e^{-\tau}\left[\cos (\tau \sqrt{4 \mathrm{St}-1})+\frac{1-2 \mathrm{St}}{\sqrt{4 \mathrm{St}-1}} \sin (\tau \sqrt{4 \mathrm{St}-1})\right]$.

This last expression indicates in particular that the period of the oscillations, $4 \pi t_{a} / \sqrt{4 \mathrm{St}-1}$, becomes large for near critical conditions corresponding to $0<\mathrm{St}-1 / 4 \ll 1$, whereas the damping of the oscillatory motion is always associated with the accommodation time $t_{a}$.

The oscillatory and nonoscillatory droplet trajectories evaluated with (63) and (64) for $\mathrm{St}=0.15$ and with (63) and (65) for $\mathrm{St}=1.0$ are shown in Fig. 4. The figure also includes the flow streamlines originating at the injection points, which differ from the associated droplet trajectories. The existence of droplet-gas slip is responsible for the accumulation of droplets as the stagnation plane is approached, with the droplet-number density becoming infinity as $z \rightarrow 0$ for $\mathrm{St}<1 / 4$. This singularity in $n$, identified in early studies of particleladen flows [117], is however of limited relevance for spray counterflow flames because nonoscillatory droplets vaporize after spending a finite time inside the hot mixing layer, with the result that they disappear at a finite distance from the stagnation plane. As seen below, vaporization also has a significant effect on the droplets traversing into the air side, which can only cross the stagnation plane a limited number of times, because during part of their oscillatory cycle they are surrounded by hot gas, resulting in droplet vaporization.

The simple droplet-trajectory problem (61) and (62), leading to the critical value $\mathrm{St}_{c}=1 / 4$ for oscillatory droplet motion, assumes a potential gas-velocity field and neglects variations of droplet radius and of gas viscosity. Effects of droplet vaporization, of gas-viscosity variation with temperature, and of departures of the gas velocity from the potential solution modify the droplet trajectory in a significant manner. As a result, it is not possible to know with certainty at the start of the computation whether a given droplet class will undergo reverse motion. In that sense, the critical value $\mathrm{St}_{c}=1 / 4$, which becomes $\mathrm{St}_{c}=1 / 8$ for the planar counterflow [122], cannot be expected to be accurate in reacting flows.

When reverse droplet motion appears for supercritical values of St, the trajectories of droplets of a given class that have been introduced at different radial locations $r_{I}$ at the same meridional plane end up crossing each other, as seen in Fig. 4. The existence of crossing trajectories implies that, for a given class of droplets injected at $z=z_{I}$, the properties achieved at a given height $z$ (notably, the droplet velocity, as shown in the figure, but also their temperature and local radius) may take different values, depending on what stretch of the oscillatory cycle is considered. As shown by Gutheil and Sirignano [75], one way of resolving this multiplicity, maintaining the selfsimilar character of the counterflow solution, is to consider in the integration the existence of different "sheets of solutions". Alternatively, this idea can be formulated in the framework of the multicontinua description by considering, as done below, that the outgoing and the returning droplets belong to different classes, so that an additional independent droplet class is added to the description every time the droplets reverse their motion. Since the number of traveling cycles is finite in the presence of vaporization, the number of additional droplet classes to be considered is always limited, and their inclusion does not tax excessively the numerical computation.

\subsection{Multicontinua formulation of counterflow spray flames}

We give below the multicontinua formulation for the self-similar solution of the steady counterflow spray flame near the stagnation plane. The formulation is equivalent to that developed by Sirignano and coworkers [75,107-109], including the modifications needed to accommodate the

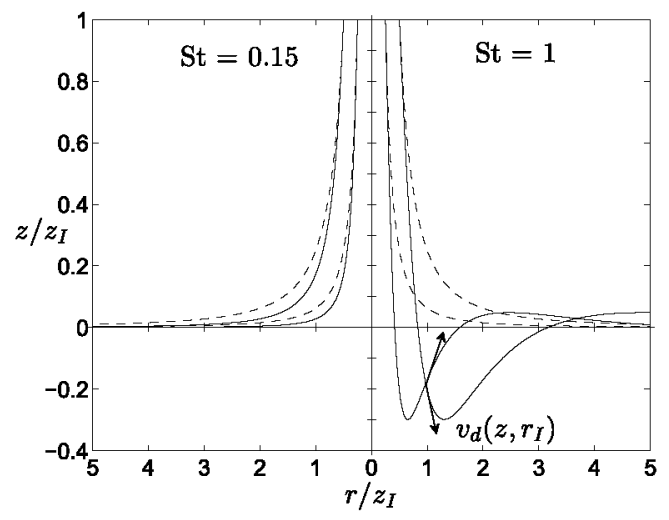

Fig. 4. Droplet trajectories obtained for $\mathrm{St}=0.15$ and $\mathrm{St}=1.0$ from evaluation of $(63)-(65)$ for $r_{I} / z_{I}=0.25$ and $r_{I} / z_{I}=0.5$. The dashed curves represent the flow streamlines $r / r_{I}=\left(z / z_{I}\right)^{-1 / 2}$. 
presence of droplets with reverse motion. The gas phase is described in terms of the radial and axial velocity components $v=A(z) r / 2$ and $u(z)$, temperature and density $T(z)$ and $\rho(z)$, and mass fractions $Y_{i}(z)$. The fuel is injected into the inert stream at a given distance $z=z_{I} \gg \delta_{m}$ from the stagnation point, forming a polydisperse spray with a $N_{c}$ different droplet classes. For each droplet class $j$, the solution at a given distance $z<z_{I}$ is given in terms of the droplet radius $a^{j}(z)$, temperature $T_{d}^{j}(z)$, radial and axial velocity components $v_{d}^{j}=$ $A_{d}^{j}(z) r / 2$ and $u_{d}^{j}(z)$, and droplet number density $n^{j}(z)$.

The problem is formulated in a nondimensional form that exhibits explicitly the dependences on the main relevant controlling parameters, including the Stokes number St and the liquid mass-loading ratio at the injection plane $\alpha$. The spray-side values of the strain rate $A_{s}$, thermal diffusivity $D_{T_{s}}=\kappa_{s} /\left(\rho_{s} c_{p}\right)$, gas density $\rho_{s}$, and gas temperature $T_{s}$ are used as scales in defining the dimensionless variables $\bar{z}=z /\left(D_{T_{s}} / A_{s}\right)^{1 / \mathcal{Z}}$, $\bar{A}=A / A_{s}, \bar{u}=u /\left(D_{T_{s}} A_{s}\right)^{1 / 2}, \bar{A}_{d}^{j}=A_{d}^{j} / A_{s}, \bar{u}_{d}^{j}=u_{d}^{j} j$ $\left(D_{T_{s}} A_{s}\right)^{1 / 2}, \bar{\rho}=\rho / \rho_{s}, \bar{T}=T / T_{s}$, and $\bar{T}_{d}^{j}=T_{d}^{j} / T_{s}$. Similarly, the spray properties at the injection location are used to define $\bar{n}^{j}=n / n_{o}^{j}$, and $\bar{a}^{j}=a / a_{o}^{j}$. In terms of this set of variables, the gas-phase problem reduces to that of integrating

$$
\begin{aligned}
\frac{\mathrm{d}}{\mathrm{d} \bar{z}}(\bar{\rho} \bar{u})+\bar{\rho} \bar{A} & =\frac{2}{3 \operatorname{Pr}} \sum_{j=1}^{N_{c}} \frac{\alpha^{j}}{\mathrm{St}^{j}} \bar{n}^{j} \bar{a}^{j} \bar{T}^{\sigma} \lambda^{j}, \\
\frac{\bar{\rho} \bar{A}^{2}}{2}+\bar{\rho} \bar{u} \frac{\mathrm{d} \bar{A}}{\mathrm{~d} \bar{z}}= & \frac{1}{2}+\operatorname{Pr} \frac{\mathrm{d}}{\mathrm{d} \bar{z}}\left(\bar{T}^{\sigma} \frac{\mathrm{d} \bar{A}}{\mathrm{~d} \bar{z}}\right) \\
& +\sum_{j=1}^{N_{c}} \frac{\alpha^{j}}{\mathrm{St}^{j}} \bar{n}^{j} \bar{\alpha}^{j} \bar{T}^{\sigma}\left(\bar{A}_{d}^{j}-\bar{A}\right)\left(1+\frac{2}{3 \operatorname{Pr}} \lambda^{j}\right),
\end{aligned}
$$

$$
\begin{aligned}
\bar{\rho} \bar{u} \frac{\mathrm{d} \bar{T}}{\mathrm{~d} \bar{z}}= & \frac{\mathrm{d}}{\mathrm{d} \bar{z}}\left(\bar{T}^{\sigma} \frac{\mathrm{d} T}{\mathrm{~d} \bar{z}}\right)-\frac{\sum_{i=1}^{N_{s}} h_{i}^{o} \dot{w}_{i}}{c_{p} T_{s} \rho_{s} A_{s}} \\
& -\frac{2}{3 \operatorname{Pr}} \sum_{j=1}^{N_{c}} \frac{\alpha^{j}}{\operatorname{St}^{j}} \bar{n}^{j} \bar{a}^{j} \bar{T}^{\sigma} \lambda^{j} \frac{\bar{T}-\bar{T}_{d}^{j}}{1-e^{-\lambda^{j}}},
\end{aligned}
$$

$$
\begin{gathered}
\bar{\rho} \bar{u} \frac{\mathrm{d} Y_{i}}{\mathrm{~d} \bar{z}}=\frac{1}{\operatorname{Le}_{i}} \frac{\mathrm{d}}{\mathrm{d} \bar{z}}\left(\bar{T}^{\sigma} \frac{\mathrm{d} Y_{i}}{\mathrm{~d} \bar{z}}\right)+\frac{\dot{w}_{i}}{\rho_{s} A_{s}} \\
+\left\{\begin{array}{l}
\left(1-Y_{\mathrm{F}}\right) \frac{2}{3 \operatorname{Pr}} \sum_{j=1}^{N_{c}} \frac{\alpha^{j}}{\mathrm{St}^{j}} \bar{n}^{j} \bar{a}^{j} \bar{T}^{\sigma} \lambda^{j}(\text { if } i=\mathrm{F}) \\
-Y_{i} \frac{2}{3 \operatorname{Pr}} \sum_{j=1}^{N_{c}} \frac{\alpha^{j}}{\mathrm{~S}^{j} j} \bar{n}^{j} \bar{a}^{j} \bar{T}^{\sigma} \lambda^{j}(\text { if } i \neq \mathrm{F})
\end{array}\right.
\end{gathered}
$$

with boundary conditions $\bar{u}+\bar{z}=\bar{A}-1=$ $\bar{T}-1=Y_{i}-Y_{i_{s}}=0$ as $\bar{z} \rightarrow+\infty$ and $\bar{A}-\sqrt{\rho_{s} / \rho_{A}}=$ $\bar{T}-\bar{T}_{A}=Y_{i}-Y_{i_{A}}=0$ as $\bar{z} \rightarrow-\infty$. Correspondingly, the evolution of each droplet class $j$ requires integration of

$$
\begin{aligned}
& \bar{u}_{d}^{j} \frac{\mathrm{d}\left(\bar{a}^{j}\right)^{3}}{\mathrm{~d} \bar{z}}=-\frac{2}{3 \operatorname{PrSt}^{j}} \bar{a}^{j} \bar{T}^{\sigma} \lambda^{j}, \\
& \frac{\left(\bar{A}_{d}^{j}\right)^{2}}{2}+\bar{u}_{d}^{j} \frac{\mathrm{d} \bar{A}_{d}^{j}}{\mathrm{~d} \bar{z}}=\frac{1}{\mathrm{St}^{j}} \frac{\bar{T}^{\sigma}}{\left(\bar{a}^{j}\right)^{2}}\left(\bar{A}-\bar{A}_{d}^{j}\right), \\
& \bar{u}_{d}^{j} \frac{\mathrm{d} \bar{u}_{d}^{j}}{\mathrm{~d} \bar{z}}=\frac{1}{\mathrm{St}^{j}} \frac{\bar{T}^{\sigma}}{\left(\bar{a}^{j}\right)^{2}}\left(\bar{u}-\bar{u}_{d}^{j}\right), \\
& \bar{u}_{d}^{j} \frac{\mathrm{d} \bar{T}_{d}^{j}}{\mathrm{~d} \bar{z}}=\frac{2 c_{p} / c_{l}}{3 \operatorname{PrSt}^{j}} \frac{\bar{T}^{\sigma} \lambda^{j}}{\left(\bar{a}^{j}\right)^{2}}\left(\frac{\bar{T}-\bar{T}_{d}^{j}}{e^{e^{j}}-1}-\frac{L_{v}}{c_{p} T_{s}}\right) \\
& \frac{\mathrm{d}}{\mathrm{d} \bar{z}}\left(\bar{n}^{j} \bar{u}_{d}^{j}\right)+\bar{n}^{j} \bar{A}_{d}^{j}=0
\end{aligned}
$$

with initial conditions $\bar{a}^{j}-1=\bar{u}_{d}^{j}-\bar{u}_{I}^{j}=$ $\bar{A}_{d}^{j}-\bar{A}_{I}^{j}=\bar{T}_{d}^{j}-\bar{T}_{I}^{j}=\bar{n}^{j}-1=0 \quad$ at $\quad \bar{z}=\bar{z}_{I}$. The above equations must be supplemented with the equation of state written in nondimensional form and with the expressions (23) and (24), needed to compute the dimensionless vaporization rate $\lambda^{j}$. Each droplet class is characterized by its massloading ratio $\alpha^{j}$ and Stokes number $\mathrm{St}^{j}$, obtained from evaluating (2) and (60) with the properties of the droplet class at the injection plane. Note that an arbitrary zero displacement of the spray stream is assumed in writing the boundary condition $\bar{u}+\bar{z}=0$ as $\bar{z} \rightarrow+\infty$. The location $\bar{z}=\bar{z}_{0}$ of the stagnation plane, where $\bar{u}=0$, is obtained as part of the integration.

The numerical computation with the multicontinua formulation requires the coupled solution of the gas and liquid phases in an iterative scheme that may start by solving the elliptic gas-phase problem Eqs. (66)-(69) incorporating an adequate guess for the distributions of the droplet properties. The resulting profiles $\bar{T}(\bar{z}), \bar{u}(\bar{z})$, and $\bar{A}(\bar{z})$ are next used in computing for each droplet class the distributions of $\bar{a}, \bar{A}_{d}, \bar{u}_{d}$, and $\bar{T}_{d}$ by integrating the characteristic equations (70)-(73) for decreasing $\bar{z}$ from $\bar{z}=\bar{z}_{I}$, while the associated number density $\bar{n}$ is obtained from (74). If the Stokes number is sufficiently small, then the value of $\bar{a}$ is seen to decay to zero at a given location, where the axial velocity $\bar{u}_{d}$ is still negative. The integration of non-reversing droplets ends at that location. By way of contrast, for droplets with sufficiently large Stokes number the value of $\bar{u}_{d}$ approaches zero before complete droplet vaporization occurs. At the turning plane $\bar{z}=\bar{z}_{t}$, where $\bar{u}_{d}=0$, the integration provides nonzero finite values of $\bar{a}=\bar{a}_{t}$, $\bar{A}_{d}=\bar{A}_{d_{t}}$, and $\bar{T}_{d}=\bar{T}_{d_{t}}$. On the other hand, the local axial-velocity distribution

$\bar{u}_{d}=\mp\left(\frac{2 \bar{T}_{t}^{\sigma} \bar{u}_{t}}{\mathrm{St} \bar{a}_{t}^{2}}\right)^{1 / 2}\left(\bar{z}-\bar{z}_{t}\right)^{1 / 2}$,

obtained from (72) in terms of the local values of the gas temperature $\bar{T}_{t}$ and gas velocity $\bar{u}_{t}$ (with the minus and plus signs corresponding to outgoing and returning droplets, respectively), can be used in (74) to show that the droplet number density diverges at the turning plane according to 
$\bar{n}=C\left(\bar{z}-\bar{z}_{t}\right)^{-1 / 2}$, where the constant $C$ is determined numerically. As can be seen, although $n$ becomes infinity at $\bar{z}=\bar{z}_{t}$, the resulting singularity remains integrable, a characteristic of the solution noted in early work [75]. To avoid the existence of multivalued functions within a given droplet class, the droplets that have turned are assigned to a newly created droplet class, whose radius, velocity and temperature are determined by integrating (70)-(73) for increasing $\bar{z}$ with initial conditions $\bar{a}=\bar{a}_{t}, \bar{A}_{d}=\bar{A}_{d_{t}}, \bar{u}_{d}=0$, and $\bar{T}_{d}=\bar{T}_{d_{t}}$ at $\bar{z}=\bar{z}_{t}$, while the associated number of droplets is obtained from (74) with a boundary value $\bar{n}=C\left(\bar{z}-\bar{z}_{t}\right)^{-1 / 2}$ evaluated near $\bar{z}=\bar{z}_{t}$.

The droplet integration procedure, including addition of a new droplet class every time the droplets turn, continues until complete vaporization is achieved (typically, after one or two turning events). The distributions of radius, velocity, temperature, and number density of all the droplet classes, i.e., those injected initially and those originated at the turning points, can be used to evaluate the droplet source terms in the gas-phase equations (66)-(69), providing upon integration updated profiles for the different gas properties, which are in turn employed to continue the calculation by recomputing the droplet evolution. The procedure is followed iteratively until convergence is achieved.

\subsection{Effects of droplet inertia on counterflow spray flames}

The formulation given above can be used to investigate different aspects of spray vaporization and combustion, including dependences on spray dilution, droplet inertia, and fuel preferential diffusion, which can be addressed by considering different values of $\alpha^{j}, \mathrm{St}^{j}$, and $\mathrm{Le}_{\mathrm{F}}$, respectively. Although the formulation can be employed in calculations of polydisperse sprays by considering different droplet classes at the injection location, only monodisperse sprays will be considered in the sample computations given below.

Because of the large value of the stoichiometric air-to-fuel mass ratio $S$, relatively small values of the spray mass-loading ratio $\alpha \sim S^{-1}$ suffice to provide the fuel needed to establish a robust counterflow spray flame. Although for these relatively small values of $\alpha$ only one-way coupling exists in the spray feed stream, in that the gas flow is not significantly affected by the vaporization and acceleration of the droplets, there still exists a substantial coupling between phases associated with the strong exothermicity of the reaction.

The two extreme solutions corresponding to chemically frozen flow and infinitely fast chemical reactions are shown in Fig. 5 for a monodisperse heptane spray counterflowing against hot air, with all parametric values being identical in both computations. As can be seen, thermal expansion modifies considerably the velocity field in the presence of combustion, including the location of the associated stagnation plane $\left(\bar{z}_{0}=-0.55\right.$ with chemically frozen flow and $\bar{z}_{0}=-3.14$ for infinitely fast combustion). Although the droplets are released at the injection point $\bar{z}_{I}=22$ with the local gas velocity, as a result of their inertia they are seen to approach the stagnation plane with a velocity whose magnitude is considerably larger than that of the gas. For the value of the Stokes number $\mathrm{St}=0.45$ used in the integrations, the incoming droplets in the upper plot, corresponding to a vaporizing spray with no chemical reaction, are seen to cross the stagnation plane, to encounter the counterflowing air stream. At their turning point, located at $\bar{z}_{t}-\bar{z}_{0} \simeq-0.505$, the axial velocity reverses sign following the local solution (75), while the droplets accumulate, as can be seen in the profiles of droplet number density. The subscripts 1 and 2 are used in the plot to indicate the properties of the outgoing and returning droplets, respectively. The large residence time in the reversing region facilitates droplet vaporization, so that the radius $\bar{a}_{2}$ is seen to decrease rapidly after the droplets turn around. Vaporization is completed before the droplets cross again the stagnation plane. The resulting fuel vapor, whose profile peaks at the turning point as a result of the
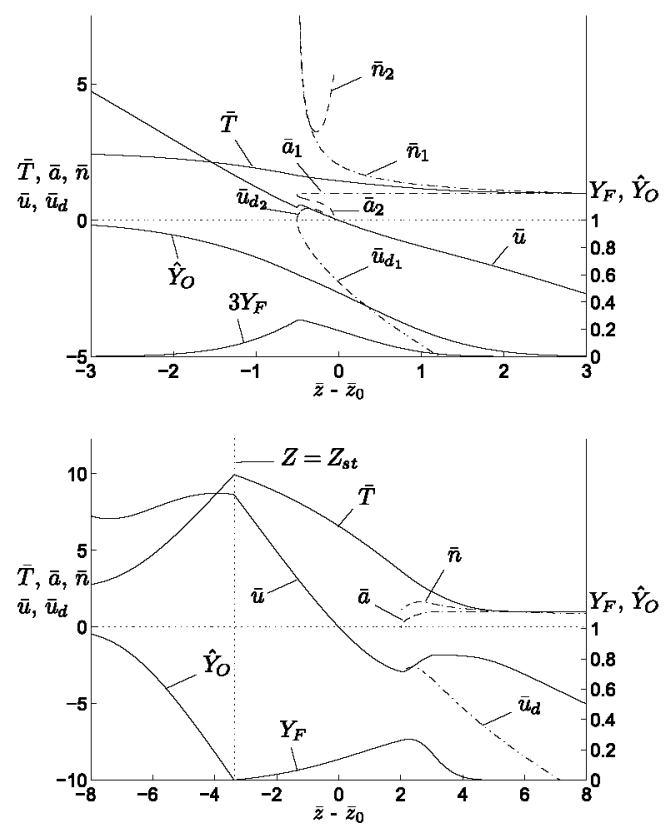

Fig. 5. Structure of a monodisperse heptane spray in a counterflow for $T_{A}=700 \mathrm{~K}, T_{s}=300 \mathrm{~K}, \alpha=0.1$, and $\mathrm{St}=0.45$. Droplets are injected at $\bar{z}_{I}=22$ with the velocity and temperature of the carrier gas. The upper plot represents inert (non-reacting) vaporization while the lower plot corresponds to infinitely fast combustion, which is computed with use made of the couplingfunction formulation introduced in Section 5 . 
local accumulation of droplets, diffuses to both sides of the mixing layer, mixing with the oxygen of the air.

The droplet behavior is different when a spray diffusion flame is present, as seen in the lower plot of Fig. 5, because the temperature increase associated with the chemical heat release enhances droplet vaporization. As a result, the droplets disappear far from the stagnation plane, at a relatively thin vaporization layer where the fuel vapor is seen to accumulate. Due to the relatively large value $S=15.2$ corresponding to heptane-air combustion, the diffusion flame, where the fuel vapor and the oxygen of the air react in stoichiometric proportions, stands away from the spray. This external sheath combustion regime is the configuration encountered in most spray counterflow diffusion flames, as was verified in numerical integrations by varying the different controlling parameters.

Computation of finite-rate effects, including critical extinction conditions, can be investigated on the basis of the Burke-Schumann computations for overall reactions strongly dependent on the temperature. Since in the extinction regime the reaction layer is thin compared with the mixing layer, its inner structure is not affected directly by the presence of the droplets; rather, as in the case of gaseous diffusion flames, it is determined at leading order by a diffusion-reaction balance. As a result, the structure of the reacting layer can be computed a posteriori, once the BurkeSchumann formulation is employed to determine the outer flame structure, including in particular the values of the peak temperature and scalar dissipation rate $D_{T}(\mathrm{~d} \tilde{Z} / \mathrm{d} z)^{2}$ at the flame. Thus, by combining the analytic results derived for gaseous diffusion flames on the basis of a one-step Arrhenius chemistry model [123] with the Burke-Schumann computations presented here one could easily evaluate critical extinction conditions for spray diffusion flames, including influences of spray dilution and droplet inertia.

Effects of preferential concentration of droplets associated with droplet-gas slip motion are investigated in Fig. 6, which represents results obtained for three different values of the Stokes number. For all three computations, the gas velocity profile shows a local minimum near the vaporization layer, that being the direct effect of the localized mass addition by droplet vaporization. The calculations employ the properties of heptane given in Table 1, with the exception of the fuel Lewis number, which is taken in this figure to be unity to focus more directly on effects of droplet inertia on flame temperature. In gaseous combustion with unity Lewis numbers of the reactants, the peak temperature is known to be the adiabatic flame temperature of the fuelair stoichiometric mixture. The adiabatic temperature resulting from burning the spray and the air in stoichiometric proportions is given by

$$
\begin{aligned}
T_{f} & =T_{s} \\
& +\frac{\left(q-L_{v}\right) / c_{p}+S\left(T_{A}-T_{s}\right)-\left(c_{l} / c_{p}-1\right)\left(T_{B}-T_{s}\right)}{S+1+1 / \alpha},
\end{aligned}
$$

with the spray properties taken at the injection plane, where the droplet are assumed to be in thermal equilibrium with the carrier gas. The value corresponding to $\alpha=0.05$, the local spray dilution at the injection plane, is indicated in Fig. 6 by a horizontal dashed line. As can be seen, the peak value of the different temperature profiles lies consistently above the adiabatic flame temperature, with increasing departures for increasing values of St. This is a result of the droplet-gas slip motion in the approaching spray stream. As can be seen in the velocity profiles on the right-hand side of the figure, the differences $\bar{u}-\bar{u}_{d}$ are more pronounced for larger values of $\mathrm{St}$, enhancing the droplet accumulation rate, an effect observed in the corresponding profiles of $\bar{n}$. As a result, for larger values of $\mathrm{St}$, the spray that reaches the vaporization layer has a higher liquid mass loading, which in turn increases the amount of fuel generated upon vaporization. The stoichiometric mixture that is burnt in the diffusion flame is therefore richer than that formed with the spray dilution corresponding to the injection conditions, leading to a higher peak temperature.

\section{Concluding remarks and open challenges}

As explained above, remarkable progress has been made over the past fifty years regarding the mathematical description of reactive spray flows by exploiting judiciously the disparity of length

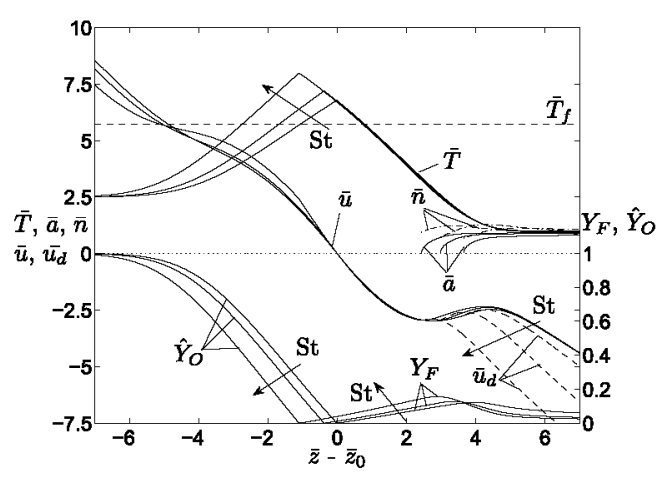

Fig. 6. Structures of counterflow Burke-Schumann flames as obtained for a monodisperse spray with St $=(0.05,0.15,0.25)$ for $\alpha=0.05, T_{A}=750 \mathrm{~K}$, and $T_{s}=300 \mathrm{~K}$. The droplets are released at $\bar{z}_{I}=15.4$ with the velocity and temperature of the carrier gas. All fuel properties are those of heptane, except for the fuel-vapor Lewis number, which is taken in these integrations to be $\mathrm{Le}_{\mathrm{F}}=1$. 
and time scales present in the problem. Reasons for the validity and limitations of the continuum description of the gas and liquid phases in the vaporization and combustion of sprays in diesel engines and liquid-fueled gas turbines have been indicated. The homogenized description can be used for the direct numerical simulation of turbulent reacting sprays and also as starting point for Reynolds-averaged representations of these flows. We have aimed to show in this paper how consideration of canonical model problems has been instrumental for the analysis of spray-combustion phenomena. In particular, we have tried to illustrate, with the examples of the evolving spray cloud and the counterflow configuration, how by the careful nondimensional formulation of the problems they can become a tool to gain understanding of the dynamics of sprays. Thus, collective effects of cloud vaporization have been investigated in a spherically symmetrical configuration, accounting for the significant separate effects of the initial liquid mass loading and the ratio of the heat-conduction time across the cloud and the time of droplet vaporization. Effects of droplet inertia on spray diffusion flames have been investigated in connection with the counterflow configuration.

Finite-rate effects, controlling the transition from the non-reacting mode to the diffusion-controlled mode, have been limited here to an Arrhenius reaction for the computation of ignition in mixing-layer configurations. The mixing-layer ignition results should be extended in future work to more realistic chemical schemes, needed for reliable predictions of finite-rate chemical effects in spray flames, including also critical conditions for strain-induced extinction, considered earlier [75]. The associated chemistry involves typically hundreds of intermediate species and a few thousand elementary reactions [124]. Chemical pretabulation in turbulent-combustion models benefits from investigations of surrogates of these complex fuels, whose chemical mechanisms are starting to require thorough uncertainty-quantification analyses as the number of kinetic parameters becomes larger $[125,126]$. Systematic chemical-kinetic reduced mechanisms based on steady-state approximations for intermediates [85] can be useful in numerical and asymptotic investigations of spray flames, including computations of pollutant emissions (see, e.g., the discussion in Section 5.4).

Despite the significant progress made in recent years, our understanding of spray-combustion phenomena is far from complete. Improved descriptions of the hydrodynamic instabilities leading to liquid-jet atomization and of droplet breakup and coalescence processes occurring in the secondary atomization region are needed. Although investigations focused on the dynamics of momentum transfer in particle-laden turbulent flows have contributed valuable understanding of the essential mechanisms leading to droplet dispersion in spray jets, including effects associated with turbulent kinetic-energy modulation by the solid phase as well preferential concentration [18] (both having important implications for subgrid-scale modeling [127-130]), the needed extension to spray turbulent combustion necessitates the additional consideration of two-way mass and thermal-energy transfer processes. More work on the effects of convection at interdroplet and droplet scales is also needed, to address influences of turbulence on homogenized descriptions and on droplet-source submodels.

The current trends that gear designs of diesel engines towards higher compression ratios promote increasing interest in spray combustion under supercritical conditions [131]. Since the liquid fuel is seldom preheated before injection, the presence of both subcritical and supercritical conditions in the combustion chamber is warranted. This requires simultaneous consideration of the liquid phase, which may contain droplets and ligaments, in addition to the gas phase and the supercritical mixture, with the spatial location of the transcritical conditions being determined by the capabilities of the hot environment to heat up the liquid fuel to its critical temperature. Clearly, the different simplifications stemming from the condition $\rho_{l} / \rho \gg 1$ do not apply to the description of the supercritical fluid. Although analyses of droplet vaporization and combustion have addressed supercritical conditions [132], including transient effects associated with shorter life times $[20,133]$, more work is needed to provide reliable droplet submodels.

Considerable advances have been made in recent years in understanding the onset and development of thermoacoustic instabilities in gaseous combustion [134-136]. Extension to spray combustion of predictive capabilities developed for gaseous combustion instabilities requires the consideration of finite inertia of the liquid phase, which responds to pressure oscillations with a characteristic delay that depends on the Stokes number $[137,138]$. In this regard, recent investigations have been carried out to model spray-flame transfer functions using numerical simulations [139] and experiments [140-142], of interest for the development of reduced-order models.

A number of modeling issues remain to be resolved. For example, the direct extension to spray combustion of flamelet approaches for the modeling of turbulent reacting flows appears to be nontrivial. Since both premixed and non-premixed flames can coexist in liquid-fueled burners $[64,69-73,143]$, the accuracy of modeling strategies based on either premixed or non-premixed flamelets is necessarily limited, so that the use of hybrid models must be considered in spray-combustion applications. In contrast to gaseous flamelets, in which the local strain rate is often 
sufficient to parametrize the entire manifold of solutions, spray flamelets display a richer parametric dependence, as can be inferred from the counterflow formulation of Section 7. On the other hand, the gas mixture fraction, which provides a one-to-one mapping between physical and mixture-composition spaces in gaseous combustion, becomes non-monotonic in spray flames because of droplet vaporization $[144,145]$. This, in turn, prevents straightforward tabulation of the flamelet variables, and introduces additional difficulties for modeling the scalar dissipation rate, which strongly departs from its gaseous counterpart [64]. To palliate this lack of monotonicity, a mixture fraction incorporating also the local liquid mass fraction may be derived, in which the vaporization source term does not appear explicitly. Nevertheless, the presence of the fuel in liquid phase in the mixture fraction requires modeling of additional terms related to the droplet-gas slip velocity and to the non-diffusive character of droplet transport [146,147]. Alternative definitions of mixture-fraction variables incorporating the liquid fuel have been proposed [148], but those appear to become more effective for numerical simulations that resolve the spray down to droplet scales including the liquid-gas interface. A different approach that parallels the treatment of gaseous combustion involves the use of a mixture fraction that satisfies a source-free transport equation [67], thereby simplifying greatly the numerical integration. Such a mixture fraction cannot be derived, however, from the original conservation equations, a shortfall of the formulation that necessarily limits its descriptive capability (e.g., the location of the stoichiometric mixture fraction in physical space is not related in any way to the flame position). Clearly, many of these modeling difficulties are not present when the droplets are so small that they heat up and vaporize very fast while following closely the gaseous streamlines, under which conditions a quasigaseous flamelet formulation based on the counterflow configuration may be used, including modified boundary conditions on the fuel side that account for the enthalpy loss required for droplet vaporization [149].

\section{Acknowledgments}

Amable Liñán's interest in spray combustion originated while collaborating at Yale University with Sandro Gomez, whose insightful input guided the initial developments of many of the ideas presented here. Much of our recent work on spray combustion has been carried out in collaboration with Forman A. Williams and Daniel Martínez-Ruiz. This work was supported by the Spanish MCINN through project
\#CSD2010-00011. Javier Urzay acknowledges support from the 2011-2012 Postdoctoral Fellowship for Excellence in Research, Ibercaja Foundation at Zaragoza (Spain).

\section{References}

[1] F.A. Williams, Combust. Flame 21 (1973) 1-31.

[2] C.K. Law, Prog. Energy Combust. Sci. 8 (1982) 171-201.

[3] G.M. Faeth, Prog. Energy Combust. Sci. 9 (1983) $1-76$.

[4] W.A. Sirignano, Prog. Energy Combust. Sci. 9 (1983) 291-322.

[5] J. Bellan, Prog. Energy Combust. Sci. 26 (2000) 329-366.

[6] H.H. Chiu, Prog. Energy Combust. Sci. 26 (2000) $381-416$.

[7] K. Annamalai, W. Ryan, Prog. Energy Combust. Sci. 18 (1992) 221-295.

[8] A. Umemura, Prog. Energy Combust. Sci. 20 (1994) 325-372.

[9] W.A. Sirignano, Prog. Energy Combust. Sci. (2014) (in press)

[10] S.K. Aggarwal, Prog. Energy Combust. Sci. 24 (1998) 565-600.

[11] X. Jiang, G.A. Siamas, K. Jagus, T.G. Karyiannis, Prog. Energy Combust. Sci. 36 (2010) 131-167.

[12] E. Gutheil, in: B. Merci, D. Roekaerts, A. Sadiki (Eds.), Experiments and Numerical Simulations of Diluted Spray Turbulent Combustion, SpringerVerlag, 2011, pp. 1-39.

[13] P. Jenny, D. Roekaerts, N. Beishuizen, Prog. Energy Combust. Sci. 38 (2012) $846-887$.

[14] S.P. Lin, R.D. Reitz, Anmu. Rev. Fluid Mech. 30 (1998) 85-105.

[15] J.C. Lasheras, E. Hopfinger, Annu. Rev. Fluid Mech. 32 (2000) 275-308.

[16] C. Dumouchel, Exp. Fluids 45 (2008) 371-422.

[17] C.T. Crowe, T.R. Troutt, J.N. Chung, Anmu. Rev. Fluid Mech. 28 (1996) 11-43.

[18] S. Balachandar, J.K. Eaton, Annu. Rev. Fhid Mech. 42 (2009) 111-133.

[19] S. Subramaniam, Prog. Energy Combust. Sci. 38 (2013) 215-245.

[20] W.A. Sirignano, Fluid Dynamics and Transport of Droplets and Sprays, 2nd Ed., Cambridge University Press, 2010.

[21] A.H. Lefebvre, Atomization and Sprays, Hemisphere Publishing Corporation, 1989.

[22] K.K. Kuo, R. Acharya, Fundamentals of Turbulent and Multiphase Combustion, Wiley and Sons, 2012.

[23] H.H. Chiu, N. Chigier (Eds.), Mechanies and Combustion of Droplets and Sprays, Begell House Publishers, 1995.

[24] K.K. Kuo (Ed.), Recent Advances in Spray Combustion, 1996, Prog. Aeronaut. Astronaut. 171.

[25] G.I. Taylor, Proc. Roy. Soc. Lond. 253 (1959) 296 312.

[26] E. Giannadakis, M. Gavaises, C. Arcoumanis, J Fluid Mech. 153 (2008) 153-193.

[27] G.I. Taylor, Seventh International Congress of Applied Mechanics 2 (1948) 280-285. 
[28] G.I. Taylor, Proc. Roy. Soc. Lond. 253 (1959) 313321.

[29] O. Desjardins, H. Pitsch, Atomization and Sprays 20 (2010) 311-336.

[30] J. Shinjo, A. Umemura, Int. J. Multiphase Flow 36 (2010) 513-532.

[31] S. Rana, M. Herrmann, Phys. Fluids 23 (2011) 091109.

[32] J. Shinjo, A. Umemura, Proc. Combust. Inst. 33 (2011) 2089-2097.

[33] V. Le Chenadec, H. Pitsch, J. Comput. Phys. 249 (2013) 185-203.

[34] E. Longmire, J. Eaton, J. Fhid Mech. 236 (1992) $217-257$

[35] H.S. Carslaw, J.C. Jaeger, Conduction of Heat in Solids, 2nd Ed., Oxford University Press, 1959, pp. 233-237.

[36] C.K. Law, W.A. Sirignano, Combust. Flame 28 (1977) 175-186.

[37] B. Abramzon, W.A. Sirignano, Int. J. Heat Mass Transfer 32 (1989) 1605-1618.

[38] C.T. Crowe, D.E. Stock, M.P. Sharma, ASME Trans. Series I-J. Fluids Eng. 99 (1977) 325-332.

[39] A. Bermúdez, J.L. Ferrín, A. Liñán, Combust. Theor. Model. 11 (2007) 949976.

[40] A. Bermúdez, J.L. Ferrín, A. Liñán, L. Saavedra, Combust. Flame 158 (2011) 1852-1865.

[41] X.Y. Zhao, D.C. Haworth, Combust. Flame (2014) (in press).

[42] P. Moin, S.V. Apte, AIAA J. 44 (2006) 698-708.

[43] N. Patel, M. Kirtas, V. Sankaran, S. Menon, Proc. Combust. Inst. 31 (2007) 2327-2334.

[44] G. Hannebique, P. Sierra, E. Riber, B. Cuenot, Flow Turbul. Combust. 90 (2013) 449469.

[45] F.A. Williams, Combustion Theory, Second ed., Benjamin Cummings, Menlo Park, CA, 1985.

[46] D. Kah, F. Laurent, L. Fréret, et al., Flow Turbul. Combust. 85 (2010) 649-676.

[47] C. Yuan, R.O. Fox, J. Comput. Phys. 230 (2011) $8216-8246$.

[48] R.O. Fox, Anmu. Rev. Fluid Mech. 44 (2012) 47-76.

[49] F.A. Williams, J. Chem. Phys. 33 (1960) 133-144.

[50] S.S. Sadhal, P.S. Ayyaswamy, J. Fluid Mech. 133 (1983) 65-81.

[51] D. Kassoy, F.A. Williams, AIAA J. 6 (1968) 19611965.

[52] M. Hermanns, High order numerical methods applied to the analysis of transport phenomena in combustion. PhD Dissertation, 2006, UPM, Madrid.

[53] J. Urzay, H. Pitsch, A. Liñán, Annual Research Briefs, Center for Turbulence Research, Stanford University/ NASA Ames, 2011, pp. 199-211.

[54] R.S. Miller, K. Harstad, J. Bellan, Int. J. Multiphase Flow 24 (1998) 1025-1055.

[55] Shashank, E. Knudsen, H. Pitsch, Annual Research Briefs, Center for Turbulence Research, Stanford University/ NASA Ames, 2011, pp. 213224.

[56] G. Del Álamo, F.A. Williams, J. Fluid Mech. 580 (2007) 219-249.

[57] S.C.R. Dennis, J.D.A. Walker, J. Fluid Mech. 48 (1971) 771-789.

[58] M. Queiroz, S-C. Yao, Combust. Flame 76 (1989) $351-368$

[59] A. Liñán, in: R. Borghi, P. Clavin, A. Liñán, P. Pelcé, G.I. Sivashinsky (Eds.) Theory of Droplet
Vaporization and Combustion, CEA-EDF INRIA 59. Editions Eyrolles, pp. 73-103.

[60] A.H. Lefebvre, Gas Turbine Combustion, Taylor and Francis, 1998.

[61] J. Heywood, Internal Combustion Engines, McGraw Hill, 1988.

[62] S.K. Marley, E.J. Welle, K.M. Lyons, Transact. ASME 126 (2004) 254-257.

[63] V.M. Reddy, D. Trivedi, S. Kumar, Combust. Sci. Technol. 184 (2012) 44-63.

[64] D. Martínez-Ruiz, J. Urzay, A.L. Sánchez, A. Liñán, F.A. Williams, J. Fluid Mech. 734 (2013) $387-423$.

[65] B. Lázaro, J.C. Lasheras, J. Fluid Mech. 235 (1997) 179-221.

[66] C. Lacour, D. Durox, S. Ducruix, M. Massot, Exp. Fluids 51 (2011) 295-311.

[67] N. Peters, Turbulent Combustion, Cambridge University Press, 2000.

[68] E. Gutheil, Combust. Sci. Tech. 105 (1995) 265 278.

[69] S.C. Li, F.A. Williams, Proc. Combust. Inst. 26 (1996) 1017-1024.

[70] S.K. Marley, E.J. Welle, K.M. Lyons, W.L. Roberts, Exp. Therm. Fluid Sci. 29 (2004) 23-31.

[71] J. Reveillon, L. Vervisch, J. Fhid Mech. 537 (2005) 317-347.

[72] M. Mikami, Y. Mizuta, Y. Tsuchida, N. Kojima, Proc. Combust. Inst. 32 (2009) 2223-2230.

[73] K. Luo, H. Pitsch, M.G. Pai, O. Desjardins, Proc. Combust. Inst. 33 (2011) 2143-2152.

[74] S.C. Li., Prog. Energy Combust. Sci. 23 (1997) 303-347.

[75] E. Gutheil, W.A. Sirignano, Combust. Flame 113 (1998) 92-105.

[76] J. Buckmaster, Prog. Energy Combust. Sci. 28 (2002) 435-475.

[77] S.H. Chung, Proc. Combust. Inst. 31 (2007) 877 892.

[78] J.B. Greenberg, L.S. Kagan, G.I. Sivashinsky, Combust. Theor. Model. 17 (2013) 1053-1066.

[79] M. Mikami, S. Miyamoto, N. Kojima, Proc. Combust. Inst. 29 (2002) 593-599.

[80] J. Arrieta-Sanagustín, A.L. Sánchez, A. Liñán, F.A. Williams, Fuel Process. Technol. 107 (2013) 81-92.

[81] V.A. Shvab, Gos. Energ. izd. M-L (1948).

[82] Y.B. Zeldovich, Zhurnal Tekhnicheskoi Fiziki 19 (1949) 1199-1210. English translation: On the theory of combustion of initially unmixed gases, NACA Tehnical Memorandum 1296 (1951).

[83] A. Liñan, The structure of diffusion flames, in: M. Onofri, A. Tesei (Eds.), Fluid Dynamical Aspects of Combustion Theory, Longman Scientific Technical, Essex, 1991, pp. 11-19.

[84] A. Liñán, Diffusion-controlled combustion, in: H. Aref, J.W. Phillips (Eds.), Mechanics for a New Millennium, Kluwer Academics, Dordrecht, 2001, pp. 487-502.

[85] N. Peters, B. Rogg, Reduced kinetic mechanisms for applications in combustion systems, in: Lecture Notes in Physics, Springer-Verlag, 1993.

[86] F.A. Williams, Proc. Combust. Inst. 24 (1992) 1-17.

[87] A.L. Sánchez, A. Lépinette, M. Bollig, A. Liñán, B. Lázaro, Combust. Flame 123 (2000) 436-464.

[88] T. Suzuki, H.H. Chiu, Proceedings of The Ninth International Symposium on Space Technology and Science, 1971, pp. 145-154. 
[89] H.H. Chiu, T.M. Liu, Combust. Sci. Technol. 17 (1977) $127-142$.

[90] M. Labowksy, D.E. Rosner, in: J. Zung (Ed.), Group Combustion of Droplets in Fuel Clouds, American Chemical Society, 1978, pp. 63-79.

[91] H.H. Chiu, Y. Kim, J. Croke, Proc. Combust. Inst. 19 (1982) 971-980.

[92] S.M. Correa, M. Sichel, Proc. Combust. Inst. 19 (1982) 981-991.

[93] S.M. Correa, M. Sichel, Combust. Sci. Tech. 28 (1982) 121-130.

[94] M. Yang, M. Sichel, AIAA paper 90-3060 (1990).

[95] G. Continillo, W.A. Sirignano, Proc. Combust. Inst. 22 (1988) 1941-1949.

[96] S.K. Aggarwal, W.A. Sirignano, Combust. Flame 62 (1985) 69-84.

[97] A. Liñán, F.A. Williams, Combust. Sci. Tech. 105 (1995) 245-263.

[98] J. Arrieta-Sanagustín, A.L. Sánchez, A. Liñán, F.A. Williams, J. Fluid Mech. 675 (2011) 435- 464.

[99] G.M. Corcos, F.S. Sherman, J. Fhid Mech. 73 (1976) 241-264.

[100] C.F. Edwards, R.C. Rudoff, Proc. Combust. Inst. 23 (1990) 1353-1359.

[101] I.K. Puri, P.A. Libby, Combust. Sci. Tech. 66 (1989) 267-292, 1989.

[102] G. Chen, A. Gomez, Proc. Combust. Inst. 24 (1992) 1531-1539.

[103] S.C. Li, P.A. Libby, F.A. Williams, Combust. Flame 94 (1993) 161-177.

[104] M. Massot, M. Kumar, M.D. Smooke, A. Gomez, Proc. Combust. Inst. 27 (1998) 1975-1983.

[105] S.C. Li, F.A. Williams, Proc. Combust. Inst. 28 (2000) 1031-1038.

[106] V.S. Santorio, A. Gomez, Proc. Combust. Inst. 29 (2002) 585-592.

[107] G. Continillo, W.A. Sirignano, Combust. Flame 81 (1990) 325-340.

[108] D. Schlotz, E. Gutheil, Combust. Sci. Tech. 158 (2000) 195-210.

[109] E. Gutheil, Z. Angew, Math. Mech. 81 (2001) S533-S536.

[110] H. Watanabe, R. Kurose, S-M. Hwang, F Akamatsu, Combust. Flame 148 (2007) 234- 248.

[111] H. Watanabe, R. Kurose, S. Komori, H. Pitsch, Combust. Flame 152 (2008) 2-13.

[112] R.J. Kee, K. Yamashita, H. Zhu, A.M. Dean, Combust. Flame 158 (2011) 1129-1139.

[113] J. Hayashi, H. Watanabe, R. Kurose, F. Akamatsu. Combust. Flame 158 (2011) 2559-2568.

[114] H. Zhu, R.J. Kee, L. Chen, J. Cao, M. Xu, Y. Zhang, Comb. Theory Model. 16 (2012) 715-735.

[115] C. Wang, A.M. Dean, H. Zhu, R.J. Kee, Combust. Flame 160 (2013) 265-275.

[116] A. Vie, B. Franzellia, Y. Gao, T. Lu, H. Wang, M. Ihme, Proc. Combust. Inst. 35 (2015), to appear.

[117] A. Robinson, Comm. Pure Appl. Math. 9 (1956) 69-84.

[118] J. Fernández de la Mora, P. Riesco-Chueca, J. Fluid Mech. 195 (1988) 1-21.

[119] G.F. Carrier, F.E. Fendell, S.F. Fink IV, C.N Folley, Combust. Flame 126 (2001) 1630-1639.

[120] I.K. Puri, P.A. Libby, Combust. Sei. Tech. 66 (1989) 267-279.

[121] N.-H. Chen, B. Rogg, K.N.C. Bray, Proc. Combust. Inst. 24 (1992) 1513-1521.
[122] J.L. Castillo, J. Macías, P.L. García-Ybarra, J. Aerosol Sci. 28 (1997) S733-S734.

[123] A. Liñán, Acta Astronautica 1 (1974) 1007-1039.

[124] Z. Luo, S. Som, S.M. Sarathy, et al., Combust. Theory Model. 18 (2014) 187-203.

[125] D.A. Sheen, H. Wang, Combust. Flame 158 (2011) 2358-2374.

[126] J. Urzay, N. Kseib, P.G. Constantine, D.F. Davidson, G. Iaccarino, Annual Research Briefs, Center for Turbulence Research, Stanford University/ NASA Ames, 2012, pp. 3-16.

[127] W.R. Michaek, J.G.M. Kuerten, J.C.H. Zeegers, R. Liew, J. Pozorski, B.J. Geurts, Phys. Fluids 25 (2013) 123302

[128] G. Jin, J. Zhang, G.-W. He, L.-P. Wang, Physica Scripta 142 (2010) 014061.

[129] P. Fede, O. Simonin, P. Villedieu, K.D. Squires, in: Proceedings of the Summer Program, Center for Turbulence Research, Stanford University/NASA Ames, 2006, pp. 247-248.

[130] M. Moreau, O. Simonin, B. Bédat, Flow Turbul. Combust. 86 (2011) 563-583.

[131] R.N. Dahms, J. Manin, L.M. Pickett, J.C. Oefelein, Proc. Combust. Inst. 34 (2013) 1667-1675.

[132] C. Sánchez-Tarifa, A. Crespo, E. Fraga, Astronaut. Acta 17 (1972) 685-692.

[133] J.S. Shuen, V. Yang, C.C. Hsiao, Combust. Flame 89 (1992) 299-319.

[134] T. Poinsot, D. Veynante, Theoretical and Numerical Combustion, R.T. Edwards, 2001.

[135] T. Lieuwen, V. Yang, Combustion Instabilities in Gas Turbine Engines, 2005, Prog. Aeronaut. Astronaut. 210

[136] H. Ying, V. Yang, Prog. Energy Combust. Sci. 35 (2009) 293, 264

[137] P. Clavin, J. Sung, Combust. Sci. Tech. 78 (1991) 265-288.

[138] C. Clanet, G. Searby, P. Clavin, J. Fhid Mech. 385 (1999) $157-197$

[139] C. Pera, J. Reveillon, Proc. Combust. Inst. 31 (2007) 2283-2290.

[140] P. Gajan, A. Strzelecki, B. Platet, R. Lecourt, F. Giuliani, AIAA J. 23 (2007) 390-397.

[141] M. De la Cruz Garcia, E. Mastorakos, Combust. Flame 32 (2008) 2397-2404.

[142] T. Providakis, L. Zimmer, P. Scouflaire, S. Ducruix, J. Eng. Gas Turbines Power 134 (2012) 111503-111508.

[143] B. Franzelli, B. Fiorina, N. Darabiha, Proc. Combust. Inst. 34 (2013) 1659-1666, 2013.

[144] C. Hollmann, E. Gutheil, Proc. Combust. Inst. 26 (1996) 1731-1738.

[145] C. Hollmann, E. Gutheil, Combust. Sci. Tech. 135 (1998) 175-192.

[146] J. Urzay, D. Martínez-Ruiz, A L. Sánchez, A. Liñán, F.A. Williams, Annual Research Briefs, Center for Turbulence Research, Stanford University/ NASA Ames, 2013, pp. 107-122.

[147] B. Franzelli, A. Vie, B. Fiorina, N. Darabiha, M. Ihme, Annual Research Briefs, Center for Turbulence Research, Stanford University/ NASA Ames, 2013, pp. 93-106.

[148] R.W. Bilger, Combust. Flame 158 (2011) 191-202.

[149] Y. Baba, R. Kurose, J. Fluid Mech. 612 (2008) 45-79. 